\title{
Viral effects on the content and function of extracellular vesicles
}

\author{
Nancy Raab-Traub ${ }^{1,2}$ and Dirk P. Dittmer ${ }^{1,2}$ \\ Abstract | The release of membrane-bound vesicles from cells is being increasingly recognized \\ as a mechanism of intercellular communication. Extracellular vesicles (EVs) or exosomes are \\ produced by virus-infected cells and are thought to be involved in intercellular communication \\ between infected and uninfected cells. Viruses, in particular oncogenic viruses and viruses that \\ establish chronic infections, have been shown to modulate the production and content of EVs. \\ Viral microRNAs, proteins and even entire virions can be incorporated into EVs, which can affect \\ the immune recognition of viruses or modulate neighbouring cells. In this Review, we discuss the \\ roles that $\mathrm{EV}$ s have during viral infection to either promote or restrict viral replication in target \\ cells. We will also discuss our current understanding of the molecular mechanisms that \\ underlie these roles, the potential consequences for the infected host and possible future \\ diagnostic applications.
}

Multivesicular bodies (MVBs). Structures below the plasma membrane that function as the central hub for the sorting of molecules into other specialized compartments, into and out of cells.
'UNC Lineberger Comprehensive Cancer Center, School of Medicine, University of North Carolina at Chapel Hill, CB\# 7295, Chapel Hill, North Carolina 27599-7295, USA.

${ }^{2}$ Department of Microbiology and Immunology, University of North Carolina at Chapel Hill, 125 Mason Farm Road, Chapel Hill, North Carolina 27599-7290, USA. nrt@med.unc.edu; ddittmer@med.unc.edu
Extracellular vesicles (EVs) are secreted from healthy, malignant and virus-infected cells. EVs are either released directly from the plasma membrane or during fusion between multivesicular bodies (MVBs) and the plasma membrane ${ }^{1,2}$. EVs that are released from MVBs are termed exosomes. Similar to EVs, viruses are released through several pathways, including the plasma membrane and through the MVB route (reviewed in REF. 3). For example, some retroviruses, such as HIV-1, assemble at the inner leaflet of the plasma membrane, whereas other retroviruses, such as Mason-Pfizer monkey virus (MPMV), first assemble in the cytoplasm before trafficking to the cell surface (reviewed in REF. 4). Some viruses are non-enveloped and do not require an envelope for infectivity, but, nevertheless, they can be incorporated into EVs for their release. For example, hepatitis A virus (HAV) was recently shown to be secreted within EVs that can potentially be transported to uninfected cells in an individual who is infected ${ }^{5,6}$. Other enteroviruses can package up to $\sim 20$ particles in a single membrane vesicle and bud without killing the host cell ${ }^{7}$. Autophagy, which is thought to provide nutrients to the cell through the digestion of intracellular organelles, can also be used for virus egress. For example, enteroviruses exit infected cells in autophagic membranes and these membranes form part of the envelope for herpesviruses, paramyxoviruses and orthomyxoviruses ${ }^{8}$. Lipidated microtubule-associated protein 1 light chain 3 (LC3), which is a marker of autophagosomes, has been detected in EVs that contain coxsackie virus. In addition, the exosome marker flotillin-1 was found in these vesicles, which suggests that picornaviruses use autophagy-related EV release as one pathway for virion exocytosis? .

EVs are thought to have an important role in virus infection and several interactions between viral and cellular components that are required for the biogenesis of EVs have been reported. Therefore, a critical comparison between virus particles and EVs may lead to an improved understanding of both virus life cycles and the functions of EVs.

Owing to their small size and similar biochemical composition, viruses and EVs have similar biophysical properties. The term exosome is used if EVs are $\leq 100 \mathrm{~nm}$ in diameter and originate from MVBs, the term microvesicle is used if the diameter is $100-1,000 \mathrm{~nm}$ and the term apoptotic body is used if the diameter is $>1,000 \mathrm{~nm}$. Similarly, viruses range in diameter from $30 \mathrm{~nm}$ for poliovirus, $120-140 \mathrm{~nm}$ for herpesviruses and 200-300 $\mathrm{nm}$ for poxviruses (reviewed in REF. 10). This similarity in biophysical properties increases the difficulty in obtaining pure populations of EVs that are not contaminated with viruses and vice versa. This makes it challenging to determine the precise composition of EVs and virions (BOX 1). The identification and characterization of virion-associated proteins have been the subject of intense study over many years ${ }^{11}$, whereas the identification of proteins that are associated with EVs has been more recent. Studies have identified key components of EVs that can be used as markers to identify and assess their purity (TABLE 1); however, it is important to recognize that not all EVs have these markers ${ }^{12}$. In the context 


\section{Biomarker}

A protein, mRNA or other small molecule that can be measured and is associated with disease outcome, either independent of treatment (prognostic) or in relation to treatment (predictive)

Chemicals, such as

polyethylene glycol or acetone

that change the availability of free solvent for proteins and

other macromolecular structures, which results in aggregation.

\section{Crowding agents}

of virus infection, viral RNAs and proteins have been found in $\mathrm{EVs}^{13-16}$, which could be the result of selective incorporation of specific RNAs and these proteins or, alternatively, reflect the total intracellular constituents. Further work is required to determine the precise composition of EVs. The development of mass spectrometers that have enhanced specificities and sensitivities compared with existing instruments will undoubtedly improve our understanding of the composition of EVs. Further work is also required to optimize the purification of EVs; therefore, careful consideration is required when attributing functional phenotypes to EVs in the context of virus infection.

Research into understanding the roles that EVs have in viral infections is driven, to a large extent, by interests in biomarker development. For example, the microRNA (miRNA) miR-122, which is incorporated into EVs during acute liver injury, could be used as a biomarker to determine the extent of damage to the liver ${ }^{17-19}$. miR-122 is the most abundant miRNA in liver

\section{Box 1 | Distinguishing EVs from virions}

The purity of any preparation of extracellular vesicles ( $E V s)$ is determined by measuring light-scatter and Brownian motion, and by electron microscopy (FIG. 2). More recently, flow cytometry-based methods have been introduced, but the small size of EVs makes them difficult to detect using conventional instruments. EVs can be isolated by size-exclusion chromatography, differential ultracentrifugation, density flotation, crowding agents, flow cytometry or affinity purification (TABLE 1); each method has specific advantages ${ }^{158}$. Size-exclusion chromatography is the best method for preserving the structure of EVs ${ }^{159-161}$, although it does not separate virions from $\mathrm{EVs}^{11,151,162}$. The use of crowding agents, such as polyethylene glycol (PEG) 3,000, followed by precipitation is the fastest way to isolate and concentrate EVs for subsequent applications; however, this approach also enriches soluble proteins and contaminants that are not a part of EVs. When profiling microRNAs (miRNAs) in EVs, it is important to consider that Argonaute (Ago)-associated miRNAs are present in serum ${ }^{163}$. These Ago-miRNA complexes also co-purify with EVs when only crowding agents are used; however, this problem can be circumvented through the use of affinity-based purification methods. Affinity purification using magnetic beads enables the high-throughput purification of EVs on robot platforms and it is able to separate EVs from viruses ${ }^{13}$. For example, EVs from B cell lymphomas are enriched for B cell surface antigens, including $C D 81$ and $C D 63$, which can be used to affinity purify $E V$ s using antibodies that bind to these proteins ${ }^{164}$. However, this approach will exclude populations of EVs that do not have the particular surface marker used for affinity purification, but that may nevertheless contribute to the biological functions of $\mathrm{EVs}^{12}$.

\begin{tabular}{llll}
\hline Method & Mechanism & Input volume & $\begin{array}{l}\text { Virion } \\
\text { co-purification }\end{array}$ \\
\hline $\begin{array}{l}\text { Differential } \\
\text { ultracentrifugation }\end{array}$ & Density and size & $35 \mathrm{ml}$ & Yes \\
\hline ExoQuick (SBI Biotech) & Precipitation & $250 \mu \mathrm{l}$ & Yes \\
\hline Total EV (Invitrogen) & Precipitation & $250 \mu \mathrm{l}$ & Yes \\
\hline PEG 2,000 & Precipitation & $250 \mu \mathrm{l}$ to $250 \mathrm{ml}$ & Yes \\
\hline CD63 magnetic beads & $\begin{array}{l}\text { Bead-based surface } \\
\text { marker }\end{array}$ & $1-35 \mathrm{ml}$ & No \\
\hline $\begin{array}{l}\text { Composite magnetic } \\
\text { beads }\end{array}$ & $\begin{array}{l}\text { Bead-based surface } \\
\text { markers }(n=5 \text { markers) }\end{array}$ & $100 \mu \mathrm{lto} 1 \mathrm{ml}$ & No \\
\hline $\begin{array}{l}\text { Size-exclusion } \\
\text { chromatography }\end{array}$ & Size-bases isolation & $100 \mu \mathrm{l}$ to $35 \mathrm{ml}$ & Yes \\
\hline $\begin{array}{l}\text { Density flotation (for } \\
\text { example, lodixanol) }\end{array}$ & Density & $35 \mathrm{ml}$ & Yes \\
\hline
\end{tabular}

cells and perhaps, owing to this abundance, it is incorporated into EVs. The correlation between the levels of miR-122 and alanine aminotransferase, an enzyme that is also released when the liver is damaged, has been established in the clinic. Therefore, miR-122 could be used as an alternative, more specific biomarker to alanine aminotransferase in the clinic. Notably, miR-122 is also required for the replication of hepatitis $C$ virus $(\mathrm{HCV})$ and is therefore an attractive drug target for the development of new therapies against $\mathrm{HCV}^{20-22}$. The success of such therapies could be determined by minimally invasive profiling of EVs and plasma miR-122 levels, as miR-122 would provide information about liver cell status in addition to viral load. The use of highly multiplexed assays that are able to detect multiple miRNAs and viruses, next-generation sequencing and mass spectrometry will help drive the research of EVs for diagnostic applications.

Studying EVs in the context of virus infection has been crucial for demonstrating the potential contribution of these vesicles to viral pathogenesis ${ }^{23}$, as EVs from virus-infected cells often transfer viral components to uninfected cells; for example, latent membrane protein 1 (LMP1) of Epstein-Barr virus (EBV) and viral miRNAs ${ }^{16,24}$. This intercellular transfer of viral cargo occurs in the absence of cell-to-cell fusion, cellular synapses or membrane nanotubes ${ }^{25-27}$, and represents the existence of a host transfer mechanism that occurs in the absence of virus spread. Roles for intercellular transport by EVs have been described; for example, in mediating cross-presentation for $\mathrm{T}$ cells ${ }^{28-30}$ and synaptic transmissions ${ }^{31}$. Many of the vesicles used during these processes have features of biogenesis and fusion mechanisms that are similar to EVs and viruses. Of note, in the context of cross-priming, the vesicles tend to stay in the immediate microenvironment, such as the lymph nodes, and are not found circulating systemically in body fluids.

In this Review, we will focus on the molecular and biological properties of EVs that are released from virus-infected cells. In addition, we consider how the EVs that are modified by viruses may either facilitate viral infection or promote resistance to immune recognition by antibodies or the inhibition of innate immunity. In particular, we will focus on human viral infections with HIV-1, HAV and HCV, and the two herpesviruses EBV and Kaposi sarcoma-associated herpesvirus (KSHV). It is in the context of these chronic, persistent and latent infections that the effects of viruses on the content and function of EVs have been studied the most.

\section{Biological roles for EVs in viral infections}

Oncogenic viruses and viruses that are able to establish long-term persistent infections have been shown to alter the content of EVs, which has been hypothesized to facilitate infection and contribute to persistence and pathogenesis. Persistent and chronic infections are characterized by low levels of virus replication and low levels of circulating virus particles $\left(\sim 10^{1}-10^{4} \text { particles per } \mathrm{ml}\right)^{3}$. By contrast, during latent infections viruses cannot be detected in the circulation. Latency has a defining role 
Table 1 | Prominent EV markers and EV-associated viral proteins

\begin{tabular}{|c|c|c|c|}
\hline Location & Marker* & Structural class & Function \\
\hline \multirow{8}{*}{$\begin{array}{l}\text { Surface } \\
\text { exposed } \\
\text { on EVs }\end{array}$} & CD9 & Tetraspanin & Cell adhesion \\
\hline & CD63 & Tetraspanin & Cell signalling \\
\hline & CD81 & Tetraspanin & Cell signalling, proliferation marker \\
\hline & $\mathrm{MHCl}$ & Histocompatibility antigen, class I & Antigen presentation \\
\hline & $\mathrm{MHC} \|$ & Histocompatibility antigen, class II & Antigen presentation \\
\hline & CD86 & $\begin{array}{l}\text { Type I membrane protein, IgG } \\
\text { superfamily }\end{array}$ & CTLA4 counter receptor B7.2 \\
\hline & FLOT1 & $\begin{array}{l}\text { Integral membrane component } \\
\text { of caveolae }\end{array}$ & $\begin{array}{l}\text { Scaffolding protein for vesicle } \\
\text { formation }\end{array}$ \\
\hline & ANXA5 & $\begin{array}{l}\text { Calcium-dependent phospholipid } \\
\text { binding protein (Annexin) }\end{array}$ & Phospholipid binding \\
\hline \multirow{4}{*}{$\begin{array}{l}\text { Internal } \\
\text { to } E V_{s}\end{array}$} & HSP70 & Heat shock protein & Mediates folding \\
\hline & HSP90 & Heat shock protein & Mediates folding \\
\hline & ALIX & PDCD6-interacting protein & ESCRT pathway \\
\hline & TSG101 & $\begin{array}{l}\text { Ubiquitylated cargo-binding } \\
\text { protein }\end{array}$ & ESCRT pathway, tumour suppressor \\
\hline \multirow[t]{11}{*}{ Virus } & EBV LMP1 & Membrane protein & Cell signalling, CD40 analogue \\
\hline & EBV LMP2a & Membrane protein & Cell signalling \\
\hline & EBV gp350 & Membrane and/or virion protein & Receptor binding \\
\hline & HIV Nef & Membrane protein & CD4 and $\mathrm{MHCl}$ downregulation \\
\hline & HSV-1 gB & Membrane and/or virion protein & Receptor binding \\
\hline & Vaccinia virus glycoproteins & Membrane and/or virion protein & Receptor binding \\
\hline & HCV (whole virus) & Virion & Infection \\
\hline & HAV (whole virus) & Virion & Infection \\
\hline & Poliovirus (whole virus) & Virion & Infection \\
\hline & Coxsackie virus (whole virus) & Virion & Infection \\
\hline & Rhinovirus (whole virus) & Virion & Infection \\
\hline
\end{tabular}

Nanotubes

Membranous protrusions that

connect adjacent cells over extended distances (up to

$100 \mu \mathrm{M}$ ) and can transfer cellular components and

viruses.

Cross-priming

The transfer of antigens from one cell to another cell, often to a professional antigenpresenting cell, that does not make the antigen; the phrase was originally coined to explain counterintuitive aspects of T cell responses.

Latent infections

The long-term presence of viral genomes (DNA or RNA) in a cell without any evidence of virion production

\section{Capsid}

Proteins that encapsulate viral genomes. Capsids are rigid, highly structured and are similar to crystals with a defined symmetry. The size, shape and symmetry of the capsid can be determined by electron microscopy and is sometimes used to classify viruses into taxa.

Endosomal sorting complex required for transport machinery (ESCRT machinery).

A multiprotein complex that is involved in the biogenesis of membrane vesicles. Viruses use the ESCRT machinery to assemble virions and bud.

ALIX, ALG2-interacting protein X; ANXA5, annexin A5; CTLA4, cytotoxic lymphocyte-associated protein 4; EBV, Epstein-Barr virus; ESCRT, endosomal sorting complex required for transport; EVs, extracellular vesicles; FLOT1, flotillin 1; gB, glycoprotein B; HAV, hepatitis A virus; HCV, hepatitis C virus; HSP, heat shock protein; HSV-1, herpes simplex virus 1; IgG, immunoglobulin G; LMP1, latent membrane protein 1; MHC, major histocompatibility complex; PDCD6, programmed cell death protein 6; TSC101, tumour susceptibility gene 101 protein. *Based on information in REFS 10,166 and www.exocarta.org. Note that not all markers are present in all EVs as demonstrated by comprehensive mass spectrometry analyses ${ }^{12}$.

in infections with herpesviruses and lentiviruses. In the case of latent herpesvirus infections, viral miRNAs can be detected within EVs at times when conventional viral load assays are negative $e^{13,16,24}$. In the case of HIV-1, the viral protein Nef has been found in EVs that circulate in infected individuals $\mathrm{s}^{32-34}$. Systemic circulation of viral proteins in EVs enables these viruses to modulate host cells without exposing viral proteins or virions to the immune system. By contrast, rapidly replicating viruses, such as Ebola virus, influenza A virus or Zika virus, accumulate to high titres $\left(10^{6}-10^{11}\right.$ particles per $\left.\mathrm{ml}\right)$ within days following primary infection. For these viruses, the viral titre is similar to the number of circulating EVs $\left(10^{10}-10^{12} \text { particles per } \mathrm{ml}\right)^{35}$. Nonetheless, it is possible that EVs that contain viral proteins or nucleic acids also modulate host cells; for example, by determining their permissiveness to infection.

The biological function of EVs in the context of viral infections can affect it in two opposing ways (reviewed in REFS 23,36-38). On the one hand, EVs can modulate recipient cells by promoting viral replication or, on the other hand, EVs can restrict viral replication by triggering host immune responses.

\section{The biogenesis of EVs}

EVs are small membrane-bound carriers of intracellular cargo that are derived from MVBs or the plasma membrane (FIG. 1). Unlike virus particles, the membranes of EVs do not enclose a structured core, such as a capsid. Specific properties of EVs define and distinguish them from other types of microvesicle ${ }^{10}$. The assembly of EVs is an active, energy-dependent and regulated process ${ }^{39,40}$. Assembly has been shown to specifically require sphingomyelinase $e^{41}$ and components of the endosomal sorting complex required for transport machinery (ESCRT machinery). The content of EVs is determined by the protein and RNA composition of the cells from which they were derived. The composition of EVs frequently reflects the relative abundance of contents in the EV-producing cell. Thus, EVs that are derived from virus-infected cells contain highly expressed 


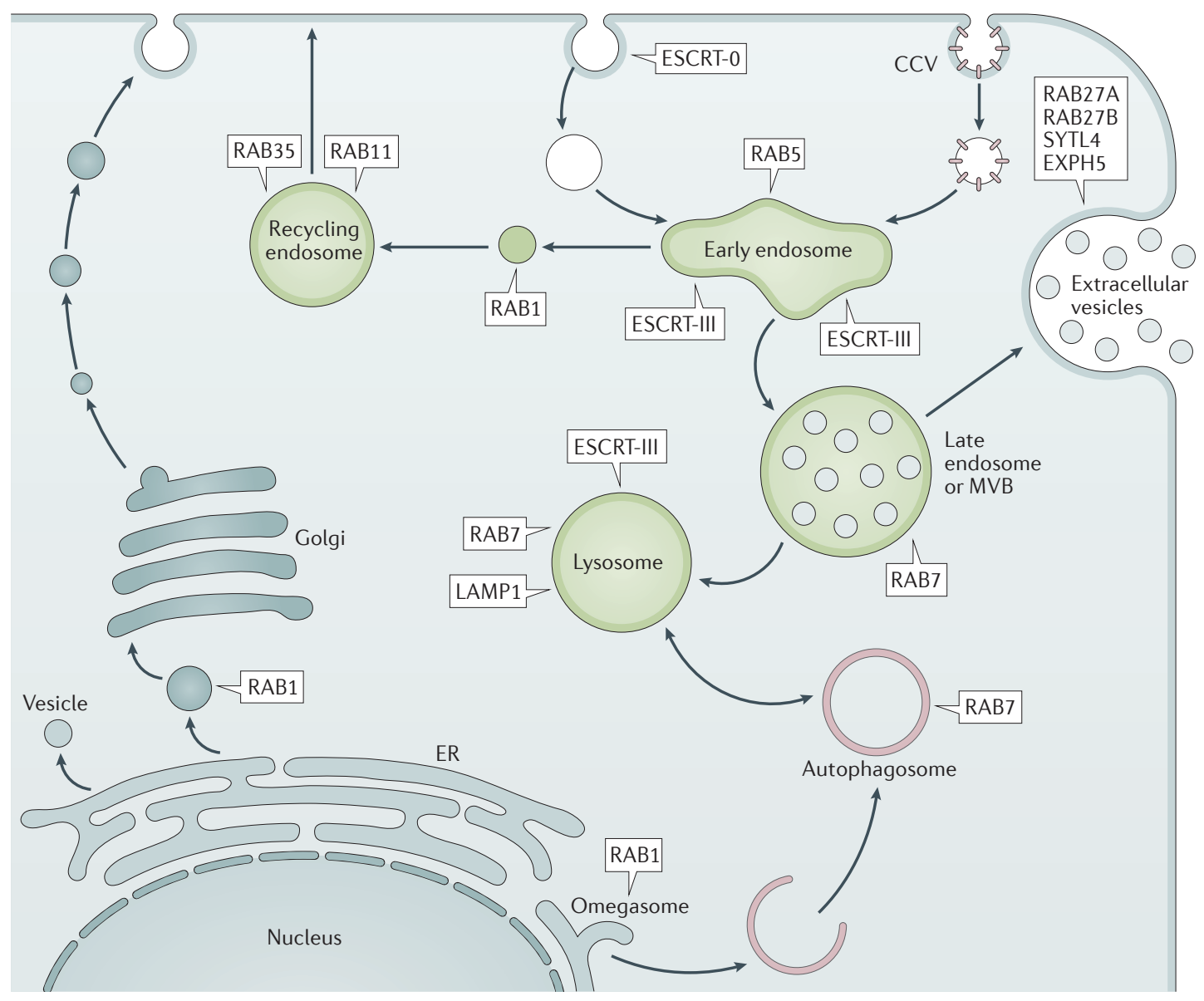

Figure 1 | Modulation of the EV biogenesis pathway during viral infection. The figure shows the main vesicular egress pathways of a cell. The late endosome or multivesicular body (MVB) sorts contents from the early endosome into either the lysosome or extracellular vesicles (EVs) for egress. The early endosome is the first step in vesicle uptake and recycling. The highlighted proteins and complexes have demonstrated functions in the biogenesis of EVs or virus maturation. CCV, clathrin-coated vesicle; ER, endoplasmic reticulum; ESCRT, endosomal sorting complex required for transport; EXPH5, exophilin 5; LAMP1, lysosome-associated membrane glycoprotein 1; SYTL4, synaptotagmin-like protein 4.

viral miRNAs ${ }^{13,16,24}$. In addition, several recent studies have provided evidence for differential loading, whereby the relative abundance of miRNAs in the EV is distinct from that of the producing cell ${ }^{16,24,42-45}$.

The budding of EVs into MVBs requires the ESCRT proteins tumour susceptibility gene 101 protein (TSG101) and ALG2-interacting protein X (ALIX; also known as PDCD6IP), which are known markers of EVs ${ }^{2}$. TSG101 and ALIX are also required for the ESCRT-dependent budding of enveloped viruses from the plasma membrane, such as herpes simplex virus 1 (HSV-1) $)^{46}$; therefore, it is difficult to determine using only these proteins as markers whether the EVs originated from MVBs or the plasma membrane. Other membrane proteins that localize to lipid rafts in the plasma membrane, including tetraspanins such as CD63 and CD81, are also enriched in EVs. Lipid raft localization involves palmitoylation ${ }^{47}$, which has been shown to be required for the incorporation of the LMP1 protein of EBV. LMP1 is enriched in lipid rafts and in EVs ${ }^{48}$. Depending on the experimental approach and cell line, other proteins, such as sortillin (SORT1), syntenin 1 (SDCBP) or syndecan 1 (SDC1), have also been found to have a role in the biogenesis of EVs ${ }^{49-51}$.

The RAB family of small GTPases regulates several steps in the trafficking of vesicles to distinct endocytic compartments and they also function in the docking of MVBs to the plasma membrane (FIG. 1). For example, the RAS-related proteins RAB11A and RAB35 function during the recycling and sorting endosomes, whereas RAB27A and RAB27B are essential for the secretion of $\mathrm{EVs}^{52}$. It is likely that some viruses modulate this process to facilitate viral entry, trafficking and egress. RAB6 and RAB7 also affect the flux between lysosomes and autophagosomes, and could contribute to determining the content of $\mathrm{EVs}^{53-55}$. Several viruses affect the expression of ESCRT and RAB GTPases to promote viral entry and egress, and are therefore likely to modulate the content of $\mathrm{EVs}^{56}$.

In addition, it is known that members of the RAB family of GTPases interact with members of the RAL family of GTPases and function during intracellular 
trafficking. In Caenorhabditis elegans, it has been shown that RAL-1 (the homologue of human RALA and RALB) regulates both the biogenesis of MVBs and the secretion of $\mathrm{EVs}^{57}$. Activated RAL- 1 associates with syntaxin 5 (SYX-5), which is a soluble $N$-ethylmaleimide-sensitive factor attachment protein receptor (SNARE) complex protein, at the plasma membrane and is required for the secretion of EVs. Interestingly, SYX5 is also required for the release of human cytomegalovirus (HCMV) and SYX4, another SNARE complex protein that has been found to regulate the release of $\mathrm{HCV}^{58,59}$. Additional SNARE complex proteins have also been shown to interact with herpesvirus glycoproteins to promote release ${ }^{60}$. These examples demonstrate that viruses co-opt the cellular vesicular transport system during egress and are therefore likely to modulate the content and secretion of EVs through similar interactions.

\section{Uptake of EVs}

Viruses use cell surface receptors to initiate fusion with the plasma membrane and are known to use specific receptors to target specific cell types. This receptor specificity determines their cellular tropism and is a distinguishing feature compared with EVs, which have the ability to enter a wider range of cell types than viruses. Using fluorescent dyes that were incorporated into EVs, it was shown that the membranes of EVs can fuse with cell membranes ${ }^{16,24,61,62}$. Most cell types that have been tested can fuse with EVs and therefore EVs can be used to deliver cargo to various cell types. This process is analogous to cationic lipid-mediated transfection approaches. In some cases, EVs use specific receptors for entry ${ }^{12,63}$ and therefore the fusion of EVs with cellular membranes can be tissue specific. For example, EVs produced by cells that are infected with EBV that express the viral glycoprotein gp350, or EVs that are engineered to express gp350, specifically target B cells that express the viral entry receptor CD21 and can block the infection of naive B cells by $\mathrm{EBV}^{61,64}$.

Heparin is involved in the initiation of host cell entry for many viruses, including retroviruses and herpesviruses ${ }^{65}$. It is a glycosaminoglycan that can bind to almost all viral envelopes. Thus, exogenous heparin or heparin beads can inhibit virion attachment through competitive binding. Cell surface-bound heparin is thought to concentrate virions before specific receptor and co-receptor engagement. Heparin is also thought to have a role in the entry of $\mathrm{EVs}^{66}$, although the fusion of EVs with the plasma membrane can occur at concentrations of heparin that block the entry of HSV-1. This provides an experimental tool to separate virion effects from EV phenotypes, as virus entry, even the entry of non-infectious or defective HSV-1 particles, can be blocked by heparin, which affirms the notion that EVs use different mechanisms for cell entry than most viruses.

Annexin A5 (and potentially other annexin family members) mediates the fusion of EVs to the plasma membrane through binding to phosphatidylserine ${ }^{67}$. Importantly, annexin A5 does not antagonize virus entry and thus can be used to distinguish EV-mediated phenotypes from virus particle or soluble moleculemediated phenotypes. Although the biogenesis of virions and EVs is similar, EVs and virions probably use different mechanisms to enter cells.

Integrins and integrin-binding matrix proteins, many of which contain a signature arginine, glycine and aspartic acid motif (RGD motif), have important roles in virus entry and their potential roles in the entry of EVs is also beginning to be understood ${ }^{68,69}$. They are thought to act as attachment factors or co-receptors that synergize with the primary receptor for virus entry. Both EBV and KSHV use integrins as co-receptors for virus entry ${ }^{70}$. RGD peptides and integrin-specific antibodies can interfere with the attachment of virions and EVs to cells ${ }^{68,70}$. They also affect signalling through the integrin homodimer and heterodimer, thus having pleiotropic effects on cell physiology. For example, the integrin-specific antibody etaracizumab is in clinical trials as anti-angiogenesis agent in non-virus associated cancers, because blocking integrin signalling can induce cell death.

Viruses have a strict requirement for cell-type specific receptors and co-receptors. These receptors engage specific envelope glycoproteins and trigger large molecular rearrangements to expose the components of the viral fusion complex. Hence, hyperimmune serum that is raised against virion components has clinical use in blocking infection; for example, in treating patients that have been exposed to Ebola virus ${ }^{71}$. By contrast, the entry of EVs is more promiscuous than viruses, and clathrindependent, caveolae-dependent, macropinocytosis ${ }^{56,62}$, phagocytosis and lipid raft-mediated uptake ${ }^{72}$ have all been shown to contribute to the entry of EVs (reviewed in REF. 63).

\section{EVs in virus infection}

Much of our knowledge about the physiological functions of systemically circulating EVs is derived from studies that have analysed their contribution to cancer metastasis $^{73-76}$ (BOX 2). It was found that EVs released from tumour cells can modulate cells in the surrounding microenvironment and drive distant metastasis by modulating stromal cell growth, cell migration, growth factor secretion and vascular permeability. A supportive microenvironment (for example, by providing growth factors and extracellular matrix attachment opportunities and increased endothelial cell permeability), is also essential for systemic virus spread. In the following sections we will review how EVs contribute to viral infection.

$\mathrm{HCV}$. HCV is a member of the Flaviviridae family. It is distinct from the arbovirus members of the Flaviviridae, as it is transmitted through blood-to-blood contact or through sexual intercourse rather than by an insect vector. $\mathrm{HCV}$ virions are enveloped and smaller than EVs $(\sim 50 \mathrm{~nm} \text { in diameter compared with } \sim 100 \mathrm{~nm} \text { for EVs })^{77}$. EVs isolated from human hepatoma cell lines infected with $\mathrm{HCV}$ were shown to contain virions ${ }^{78,79}$. Some of the subgenomic HCV RNA co-localizes with CD81 and CD63 (markers of EVs). The E2 protein of HCV was also found to colocalize with CD81 in EVs, and EVs were 


\section{Box $2 \mid$ EVs in cancer}

Approximately $20-30 \%$ of all cancers are associated with viral infections, and many features of cancer are also features of viral infection, such as dissemination (metastasis in cancer), uncontrolled DNA replication and metabolic perturbation (for example, glycolysis and nucleotide biogenesis). Therefore, insights that are gained from the study of the physiological phenotypes of extracellular vesicles (EVs) in the context of cancer and metastasis could also be relevant to understanding the role of EVs in viral persistence ${ }^{165}$. EVs have been extensively studied in cancer research, as they are released in high levels from tumour cells ${ }^{73-76}$. The contents of EVs that are released from tumour cells mirror the contents of the producing cells. Therefore, these EVs are useful biomarkers, and the identification and characterization of EVs in body fluids from 'liquid biopsies' provide a non-invasive diagnostic and prognostic indicator for the development and progression of cancer ${ }^{13,131}$. The identification of proteins such as $\mathrm{p} 53$, epidermal growth factor (EGF) and fibroblastic growth factor (FGF) in EVs suggests that EVs also have a direct role in oncogenesis. EVs are able to transfer proteins from malignant cells to neighbouring or to distant cells, which can promote cancer growth through potential effects on the microenvironment, inhibition of anticancer immune responses and the induction of angiogenesis. Interestingly, EVs have also been shown to facilitate metastasis by inducing epithelial-to-mesenchymal transition (EMT) and other changes in the target microenvironment ${ }^{73-75}$.
Cytopathic effect

Changes in host cells that are

caused by virus infection. found to transport viral RNA to uninfected cells ${ }^{79-81}$. $\mathrm{CD} 81$ is the dominant co-receptor for $\mathrm{HCV}^{82-84}$; therefore, it is likely that $\mathrm{HCV}$ virions are incorporated into EVs through their interaction with CD81. HCV RNA that is transmitted by EVs induces an innate interferon- $\alpha$ (IFNa) response in neighbouring dendritic cells (DCs). This is in contrast to natural HCV infection, which also delivers viral RNA to cells but downregulates Toll-like receptor (TLR) and RIG-I-like receptor (RLR) signalling through the action of the viral NS3/4 protease. Therefore, by specifically transporting viral RNA but not viral proteins, which antagonize innate immunity, EVs may provide a protective function for uninfected cells in the immediate microenvironment during infection.

Systemically circulating EVs that contain HCV virions may have a pro-viral role, as HCV was shown to spread in the presence of neutralizing antibodies ${ }^{78,85}$. This spread may be facilitated by the masking of viral proteins in the EVs (FIG. 2a). Such a strategy of host evasion, in which persistent viruses, such as HCV and more prominently HAV (see below), escape the evolutionary selection pressure of neutralizing antibodies by being incorporated in EVs, may be widespread. The carriage of $\mathrm{HCV}$ virions in EVs also suggests that $\mathrm{HCV}$ could enter additional cell types, in addition to hepatocytes, through EV-mediated fusion, in which case infection would not be dependent on the expression of a specific viral receptor.

$\boldsymbol{H A V}$. HAV, which is a non-enveloped picornavirus, is the cause of acute enterically transmitted hepatitis and replicates efficiently in the liver. HAV rapidly replicates in susceptible cells and virus particles are subsequently released; however, no cytopathic effect has been observed. RNA-containing proteinaceous particles (with a density of $1.22-1.28 \mathrm{~g} \mathrm{ml}^{-1}$ ) represent the major form of HAV in faeces ${ }^{6}$. HAV can be neutralized by antibodies that are elicited by current HAV vaccines. It has been shown that HAV can be released from cells in host-derived membranes at a density of $1.06-1.10 \mathrm{~g} \mathrm{ml}^{-1}$ (REF. 5). These HAV-containing EVs are infectious and circulate in the blood of infected individuals, whereas nonenveloped viruses have only been found in faeces ${ }^{6}$ (FIG. 2b). Two other picornaviruses, coxsackie B virus and enterovirus 71 (EV71), have also been found in $\mathrm{EVs}^{7,86,87}$.

Two hypotheses have been formulated to explain the importance of EV-encapsulated picornaviruses as a biological mechanism rather than a side product of cellular inefficiency ${ }^{6}$. First, the envelopment of HAV by host membranes may expand the tropism of the virus, as EV surface proteins now engage the target cells rather than the viral surface proteins. This may provide an additional route for the spread of HAV within the liver and systemically to distant organs, such as the spleen, and lymph nodes, which function to filter and survey systemic fluids ${ }^{3}$. Second, the acquisition of a host membrane by HAV demonstrates that some non-enveloped viruses can acquire an envelope that is devoid of viral transmembrane proteins and thus provides evidence for an alternative egress pathway. This suggests that egress mediated by EVs would be distinct from the normal egress route. The acquisition of a host-derived membrane could also affect the recognition of HAV capsid proteins by antibodies and may enable persistence and spread in the presence of neutralizing antibodies.

HIV-1. HIV-1 is a human retrovirus that contains an RNA genome and acquires its envelope from the cellular plasma membrane, where it buds from areas that are enriched in the viral Gag protein. After a period of acute replication $\left(10^{5}-10^{7}\right.$ virus particles per $\left.\mathrm{ml}\right), \mathrm{HIV}-1$ establishes clinically latent infection, which slowly progresses to AIDS in untreated individuals, as the virus depletes $\mathrm{CD} 4^{+} \mathrm{T}$ cells. It is thought that during clinical latency ( $\leq 5 \times 10^{3}$ virus particles per $\mathrm{ml}$ in plasma), HIV directly and indirectly modulates the immune system, leading to chronic pathology. Many of these indirect effects on the immune system are also observed in patients who are on antiretroviral therapy. Various hypotheses for this phenomenon have been proposed, including a role for EVs. In addition, soluble viral proteins, such as Tat, as well as secondary events to immunocompromise, such as extended lipopolysaccharide (LPS) translocation, may contribute to this phenotype ${ }^{88,89}$ (reviewed in REF. 36). The assembly of HIV-1 and the biogenesis of EVs have many similarities ${ }^{90-92}$. These similarities impair the biochemical and physical separation of EVs and virions, which include mature, immature and even defective particles. The molecular pathogenesis of HIV-1 suggests potential EV-mediated effects on neighbouring cells (FIG. 2C), as the virus kills more abortively infected or uninfected T cells than infected cells ${ }^{93-96}$. Cells infected with HIV may also release EVs that have trapped HIVvirions $^{94-97}$.

More importantly, HIV encodes several accessory proteins, such as virion infectivity factor (Vif) ${ }^{98}$, that interfere with the cellular antiviral response. These pro-viral proteins may be carried by EVs to prime neighbouring cells to promote infection in what is known as the 'Trojan horse' hypothesis ${ }^{23,36}$. Conversely, 
a HCV

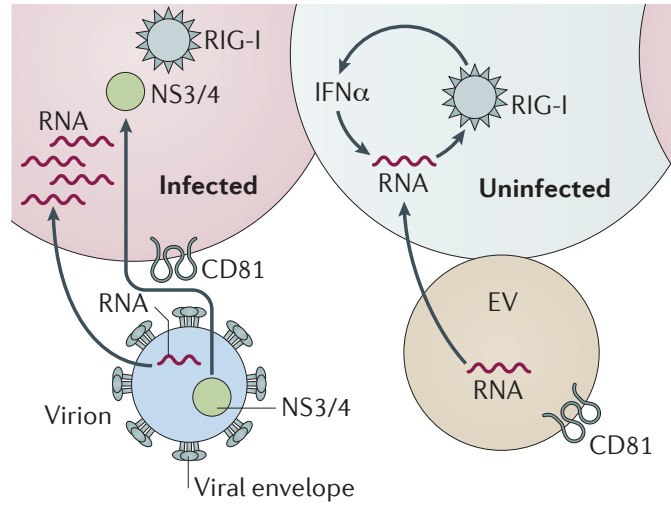

c HIV

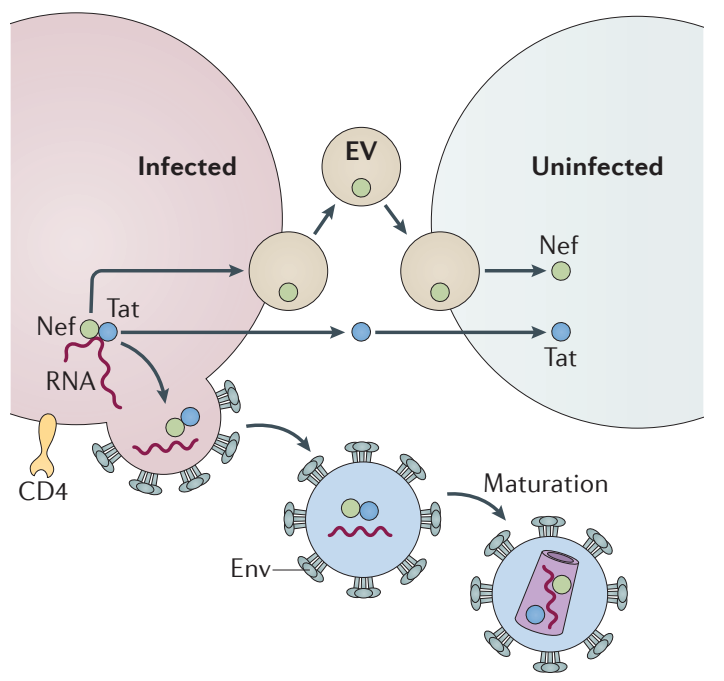

\section{d EBV}

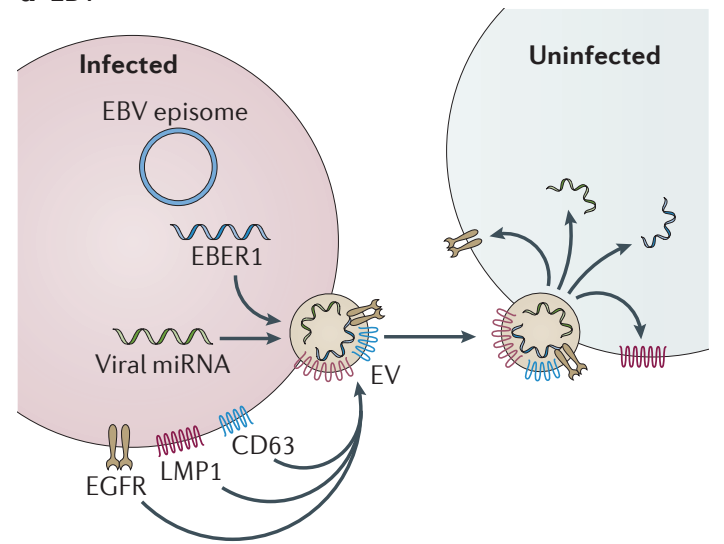

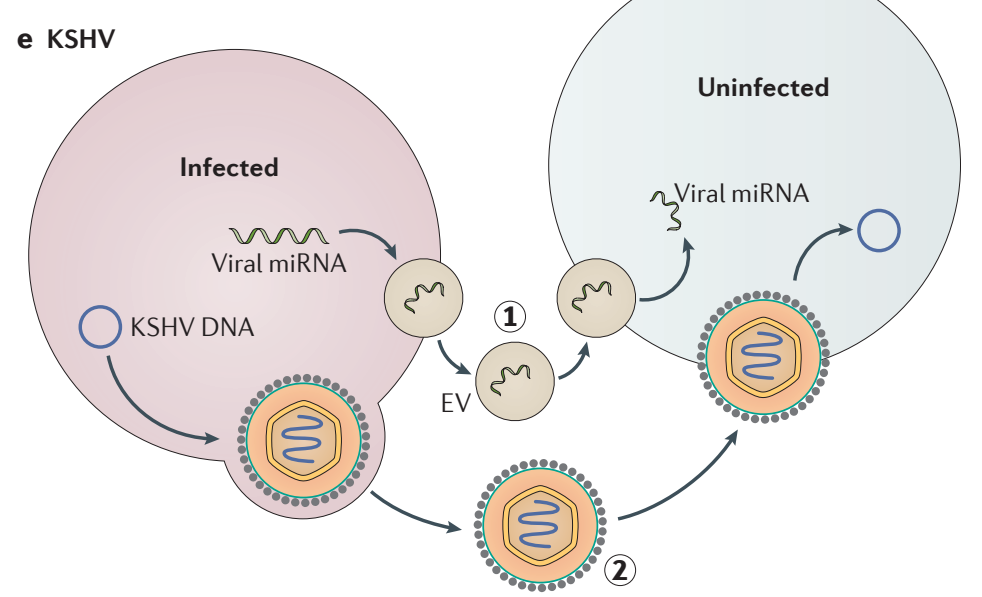

Figure 2 I Interactions between viruses and EVs. For each virus (hepatitis $\mathrm{C}$ virus (HCV), hepatitis A virus (HAV), HIV-1, Epstein-Barr virus (EBV) or Kaposi sarcoma-associated herpesvirus (KSHV)), a virion-dependent transfer and extracellular vesicle (EV)-dependent transfer step are shown. Only in the case of HCV (part a) and HAV (part b) have entire virions been identified within EVs. For the other viruses, individual RNAs or proteins have been detected in EVs. During infection with HIV (part c), Nef can be incorporated into EVs and subsequently transported to uninfected cells. The soluble HIV Tat protein can be transported to uninfected cells without being incorporated into EVs. In the case of EBV (part d), cellular and viral proteins, and cellular and viral RNAs, are transported by EVs from infected cells to uninfected cells. In the case of KSHV (part e), a temporal model is shown, whereby viral microRNAs (miRNAs) are transported by EVs before infection (step 1) and may thus prime the recipient cell for infection (step 2). EBER1, Epstein-Barr virus encoded RNA 1; EGFR, epidermal growth factor receptor; IFNa, interferon- $\alpha$; LMP1, latent membrane protein 1; NS3/4, non-structural protein 3/4; RIG-I, retinoic acid-inducible gene-I protein; VPg, viral protein genome-linked. 
cellular antiviral factors that are carried by EVs could facilitate an antiviral response in neighbouring cells ${ }^{99}$. In addition, EVs that are packaged with viral proteins may function to introduce these proteins and activate $B$ cells and T cells through the endosomal presentation of proteins, a process termed cross-priming, rather than endogenous synthesis and presentation by MHC molecules. Thus, these bystander immune cells would become activated without actual viral infection ${ }^{100,101}$.

It is known that infection with HIV alters the repertoire of miRNAs in infected cells ${ }^{102-104}$ and considering that miRNAs can be packaged into EVs ${ }^{13}$, the EVs from cells infected with HIV may contain distinct miRNAs compared with EVs from uninfected cells. In the context of AIDS-defining malignancies, such as Kaposi sarcoma, EVs isolated from patients who are infected with HIV have distinct miRNAs profiles before and concurrent with lesion development ${ }^{13,105}$. Initially, HIV was thought to encode miRNAs ${ }^{106}$, but these observations have since been questioned ${ }^{107,108}$. It is becoming clear that miRNAs that have Drosha-dependent hairpinlooped precursors are not the only small RNAs that can be transcribed from the genomes of RNA viruses (including retroviruses) ${ }^{109-111}$ and some of these RNA species have been found in EVs ${ }^{112}$. The potential roles that miRNAs (as defined by their biogenesis and structural composition), or other small RNAs that do not fit the classical definition of miRNAs, have in the function of EVs is an active area of investigation that is likely to reveal new properties that affect viral infection and pathogenesis ${ }^{107,108,113}$.

EVs from cells that are infected with HIV contain the viral protein $\mathrm{Nef}^{32-34}$. In addition, soluble Tat protein circulates in interstitial spaces, blood and mucosal barriers, and can carry out biological activities, such as promoting angiogenesis and endothelial cell reprogramming $89,114-116$. Soluble Tat has a fusogenic peptide sequence that enables its efficient uptake into cells ${ }^{117,118}$. As the HIV Tat protein does not need to be incorporated into EVs to enter cells, the biological relevance of its incorporation into EVs remains unclear. Nef associates with membranes and with proteins of the vesicular trafficking system, such as ALIX and others ${ }^{119,120}$. Nef can be incorporated into EVs and may modulate the contents of EVs, including miRNAs ${ }^{32-34,121}$; however, some researchers have contested these findings, as the level of Nef in EVs is at the limit of detection ${ }^{122}$. In summary, HIV provides an example of how latent viruses may use EVs to maintain a susceptible host environment over long periods of time, during which virus replication is minimal.

$\boldsymbol{E} \boldsymbol{B V}$. EBV, which is a gammaherpesvirus, is a major human pathogen and was the first human tumour virus to be identified ${ }^{3}$. EVs that contain viral proteins were first shown to be produced from B cells that were infected with EBV. The major EBV oncoprotein, LMP1, was identified in EVs secreted from cell lines that were infected with $\mathrm{EBV}^{123}$. LMP1 is required for B lymphocyte transformation (reviewed in REF. 124). The incorporation of this protein into EVs has not only been demonstrated in B cells and epithelial cells cultured in vitro but has also been detected in exosomes in the serum of patients with EBV-associated tumours and in serum from mice that have nasopharyngeal carcinomas (NPCs) ${ }^{16,125}$. The interaction between LMP1 and the tetraspanin CD63 may contribute to the selective incorporation of LMP1 into EVs ${ }^{48,126}$, similar to the selective palmitoylation of LMP1 (REF. 47). In addition, LMP1 is known to localize to lipid rafts and such rafts are present in the membranes of EVs ${ }^{127}$. It is possible that the presence of LMP1 in lipid rafts may contribute to the enrichment of LMP1 in MVBs and subsequent enrichment in EVs.

EVs secreted from B cells that contain LMP1 inhibit T cell proliferation and natural killer (NK) cell cytotoxicity ${ }^{123,128}$. EVs that are secreted from NPC cells infected with EBV also contain galectin 9, which is thought to contribute to these immunosuppressive effects ${ }^{128-130}$. It has long been known that NPC tumours infected with EBV are infiltrated with T cells that are seemingly non-functional, as they do not kill tumour cells or impair tumour growth. This lack of activity may reflect the abundant secretion of EVs during infection with $\mathrm{EBV}$, thus representing another viral immune evasion strategy.

Importantly, EVs that contain LMP1 have been shown to deliver activated signalling proteins into uninfected cells ${ }^{131}$. This potentially important feature of EVs was revealed in studies that showed that epidermal growth factor receptor (EGFR), which is highly induced by LMP1, was also abundant in EVs that contained LMP1 (REF. 16). LMP1 has also been shown to increase the level of phosphoinositide 3-kinase (PI3CA) in lipid rafts and EVs. The delivery of LMP1, EGFR and PI3CA through EVs induced growth-stimulating signalling pathways in recipient cells, including the activation of the phosphoinositide 3-kinase (PI3K) target, serine/threonine kinase AKT1, and extracellular signalrelated kinase 1 (ERK1; also known as MAPK3) ${ }^{131}$. An early study revealed the increased incorporation of fibroblast growth factor 2 (FGF2) from cells that expressed LMP1 into EVs, which could potentially affect the tumour environment through the direct stimulation of the growth of infected cells or supporting stromal cells ${ }^{132}$. In addition, it was demonstrated that LMP1 activates hypoxia-inducible factor $1 \alpha$ (HIF1 $\alpha$ ), which is also transferred by EVs into recipient cells and can activate various targets ${ }^{133}$. HIFla is the major transcriptional regulator under hypoxic conditions, which are characteristic of many tumours, and could promote the survival of tumour cells in an anoxic environment. An important target of HIF1 $\alpha$ is vascular endothelial growth factor 1 (VEGF1), which induces angiogenesis. Thus, through the transfer of EVs, EBV can affect the growth of neighbouring cells.

It is also known that in tumours that are caused by infection with EBV, not all cells express LMP1. Therefore, the secretion and uptake of LMP1 into cells that do not express it, could affect the growth of additional tumour cells. This may be particularly important in the pathogenesis of NPC, in which not all cells express detectable levels of LMP1.
Small, narrow spaces between tissues that are typically filled with interstitial liquid. 
LMP1 may also modulate the selective sorting of proteins into the exosomal pathway, which suggests that EBV manipulates these pathways for intercellular communication. The possibility of LMP1-mediated specific effects on the content of EVs was revealed from quantitative proteomics and 2D gel analysis of EVs purified from $B$ cell lines that were uninfected, or infected with EBV, KSHV or both viruses ${ }^{131}$. Analysis of LMP1-positive versus LMP1-negative cell lines revealed 217 protein spots with significantly different expression $(P<0.05)$. Principal component analysis to identify the distinguishing features among the EVs from these different cells lines revealed that LMP1 was a major determinant of the variance between samples. This strongly suggested that LMP1 had an effect on the exosomal protein content and provided additional evidence for specific viral effects on this process.

Spectral counting analysis also indicated that both KSHV and EBV had distinct effects on the contents of EVs and that these effects reflected cellular changes that occur in infected cells ${ }^{131}$. Gene ontology pathway analyses of proteins that were identified in EVs derived from infected cells predicted that EVs from cells infected with EBV and KSHV modulate cell death and survival, ribosome function and protein synthesis. Analyses of the contents of EVs from infected cells also indicated that EVs from cells infected with KSHV could affect cellular metabolism and that EVs from cells infected with EBV could activate cellular signalling mediated by integrins, actin, IFN and nuclear factor- $\kappa \mathrm{B}(\mathrm{NF}-\kappa \mathrm{B})$, through the transfer of crucial regulatory proteins in these pathways.

A novel finding was that EBV-encoded miRNAs

Principal component analysis

A statistical method used to uncover relationships defined by $10-1,000$ or more correlated variables, which identifies the factors that contribute to variability. Typically, the first 3-5 principal components are composed of the variables that have the greatest explanatory power.

Spectral counting

A method that determines the relative presence or absence

of a peptide in a pair of samples analysed by mass spectroscopy

Primary effusion lymphoma A diffuse large $B$ cell

lymphoma that is caused by Kaposi sarcoma-associated herpesvirus.

\section{Paracrine}

Affecting the physiology of neighbouring cells without cell-to-cell contact, typically through cytokines or growth factors. If the growth factors act on the same cell type from which they originate the process is called an autocrine loop. were also detected in EVs that were secreted from cells infected with EBV and that these viral miRNAs could then be transferred to uninfected recipient cells ${ }^{16,24}$ (FIG. 2d). The viral miRNAs were shown to specifically decrease previously identified viral miRNA targets, thus providing evidence of the functional delivery of miRNAs through $\mathrm{EVs}^{24}$. This transfer also probably occurs in vivo, as uninfected B cells that were isolated from patients with NPC contained viral miRNAs. Interestingly, EVs from NPCs have distinct patterns of EBV miRNA abundance compared with the intracellular levels in the producing cells ${ }^{16}$. This observation supports the hypothesis that there is selective sorting of specific miRNAs into EVs.

In addition to viral proteins and miRNAs, 5'pppEBER1, which is a small non-coding viral RNA, has also been found in EVs secreted from cells infected with $\mathrm{EBV}^{134}$. EBER1 is the most abundant viral RNA in infected cells and 5'pppEBER1 enhances the immune function of dendritic cells. This unusual finding indicates that EVs may contribute to autoimmune diseases, such as systemic lupus erythematosus, that have been linked to infection with $\mathrm{EBV}^{3}$. Overall, these findings suggest that EBV modulates EVs to secrete viral and cellular proteins and miRNAs that probably contribute to intercellular communication and affect the function of uninfected cells. Modulating the content of EVs could be important for affecting tumour environments by inducing cell growth, promoting angiogenesis and inhibiting immune cell function, and also for potentiating metastasis.
KSHV. KSHV causes Kaposi sarcoma and various hyperplastic and neoplastic B cell disorders, such as primary effusion lymphoma (PEL). PEL usually presents with liquid effusions without tumour masses in serous body cavities. Cell-free primary PEL fluid is highly enriched in tumour-derived EVs ${ }^{13}$. Primary KSHV infection is asymptomatic in a healthy host and results in lifelong latency. In rare cases, immune reconstitution inflammatory syndrome follows, which is associated with severe disease flares and clinical symptoms that are consistent with infection and inflammation. Common to all Kaposi sarcoma pathology is neo-angiogenesis and the infiltration of the environment that surrounds the tumour cells with uninfected non-transformed host cells, such as endothelial cells and macrophages. Aside from haemangioma, Kaposi sarcoma is the most angiogenic cancer, and therefore the study of KSHV can be useful for understanding the interactions between EVs and endothelial cells.

$\mathrm{KSHV}$ angiogenesis is driven by paracrine effectors, such as soluble cytokines and the growth factors VEGF1 and platelet-derived growth factor 1 (PDGF1), PDGF2, PDGF3 and PDGF4 (REF. 135). Initially, the paracrine drivers of Kaposi sarcoma were thought to be only soluble cytokines (for example, VEGF and PDGF or interleukin-6 (IL-6)). More recently, EVs have been shown to mediate some of these phenotypes (such as endothelial cell remodelling, migration and proliferation), independent of IL-6 (REF. 13), as PEL and Kaposi sarcoma tumour cells release large quantities of EVs, which drive endothelial cell proliferation and invasion in the presence of neutralizing antibodies to IL-6 (REF. 13).

KSHV encodes several viral miRNAs ${ }^{136,137}$, which constitute up to $50 \%$ of all miRNAs in infected cells ${ }^{138}$. Viral miRNAs and mRNAs have been detected in virion preparations of almost all herpesviruses ${ }^{14,15,139}$. Viral miRNAs are also readily detected in EVs derived from Kaposi sarcoma tumours ${ }^{140}$. The levels of viral miRNAs in EVs are 10-100 fold higher than those reported for virion-associated miRNAs. Thus, owing to the similar biophysical characteristics of virions and EVs, it is unclear whether reports of virion-associated miRNAs reflect contamination by EVs.

Several lines of evidence have attributed phenotypes that were previously attributed to miRNAs incorporated into virions to miRNAs incorporated into EVs. These include the biochemical separation of EVs by positive selection on anti-CD63 beads (a marker of EVs; FIG. 3), as well as genetic approaches, such as the detection of KSHV miRNA-containing EVs in KSHV-miRNA transgenic mice, which cannot produce the virus ${ }^{13}$. This suggests that during KSHV latency viral miRNAs can be incorporated into EVs through the same host cellular pathways that load host miRNAs into EVs. A large proportion of host miRNAs are also incorporated into systemically circulating $\mathrm{EVs}^{13}$. Thus, the identity and abundance of miRNAs in EVs represents a snapshot of their cellular origin. A more active model hypothesizes that viral miRNAs and proteins are specifically and differentially loaded into $\mathrm{EVs}^{16,24,42-45}$. 


\section{REVIEWS}

a
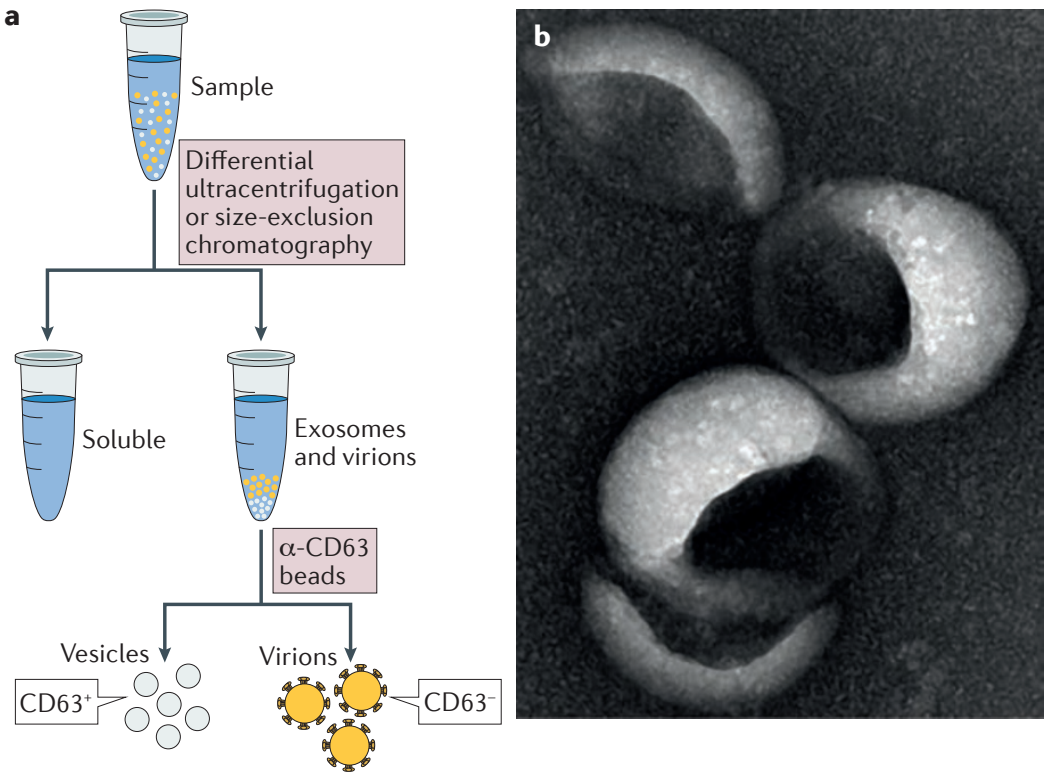

Figure 3 | Isolation of EVs and virions. a | The separation of extracellular vesicles (EVs) from virions by positive selection. First, particles are concentrated by ultracentrifugation; most virions co-purify with EVs under these conditions. Next, EVs are immunopurified using antibodies that bind to proteins present on the surface of EVs. Virions, which do not contain these proteins, are left behind in the column flow-through. Virions can also be purified by using an antibody that binds to a virion protein (not shown). b | Electron micrograph of EVs that are derived from a tumour cell line.

Infection with KSHV changes many aspects of the physiology of the infected cell, including lipid metabolism and presumably vesicle biogenesis. The protein composition of EVs and their secretion are modulated during viral reactivation from PEL cell lines ${ }^{16}$, although KSHV proteins have not been detected in $\mathrm{EVs}^{131}$. The replication and maturation of KSHV are much slower than with other viral infections ${ }^{3}$. In culture models of KSHV, the number of infectious virions is approximately $10^{5}$ copies per $\mathrm{ml}$, compared with $10^{7}$ copies per $\mathrm{ml}$ for EBV or $10^{9}$ copies per $\mathrm{ml}$ for flaviviruses ${ }^{141}$. In patients with Kaposi sarcoma, the viral titre ranges from $10^{3}-10^{5}$ copies per $\mathrm{ml}$, whereas EBV or HCMV titres exceed $10^{6}$ copies per $\mathrm{ml}$ in patients, and viral titres during hepatitis virus infections range from $10^{5}-10^{9}$ copies per $\mathrm{ml}$. In the case of KSHV, we speculate that preventing the accumulation of highly immunogenic virions, and instead using host EVs to distribute viral miRNAs to neighbouring cells, represents a novel strategy to persist in the host (FIG. 2e). In this model, miRNAs in EVs provide an evolutionary advantage for virus spread by priming neighbouring cells for infection. KSHV infects and replicates primarily in endothelial cells; these need to be attracted and re-programmed to migrate towards the initially infected cell. PEL-derived EVs confer this property to uninfected endothelial cells in culture through the transfer of viral miRNAs or protein-coding RNAs ${ }^{142,143}$. In summary, KSHV represents another example by which a virus can modulate the host local environment through $\mathrm{EV}$ reprogramming. Interfering with the release of EVs during infection with KSHV may therefore have the potential to limit virus spread and/or pathology.
Alphaherpesviruses and betaherpesviruses. Alphaherpesviruses, such as HSV-1, and betaherpesviruses, such as HCMV, also influence the biogenesis pathways of EVs and use these pathways for egress ${ }^{144-146}$. Similar to the biogenesis of EVs, the maturation of HCMV virions is dependent on the ESCRT machinery and virions contain cellular markers that are associated with $\mathrm{EVs}^{147,148}$. Some of the observed systemic and biological phenotypes that are associated with infection with HSV-1 and HCMV may be the result of massive re-programming of MHC I and MHC II trafficking from the secretory pathway. HCMV replicates in various cell types, including endothelial cells that line the blood and lymphatic vasculature, and can cause graft rejection in organ transplantation. EVs that are released from endothelial cells infected with HCMV can exacerbate allogeneic graft rejection ${ }^{149}$.

More recently, it was shown that the immune sensor stimulator of IFN genes (STING) is incorporated into EVs that are secreted from cells infected with HSV-1 and that the STING ligand cyclic GMP-AMP synthase (cGAMP) is present in EVs that are released from cells infected with murine cytomegalovirus ${ }^{150,151}$. The cGAMP nucleotide triggers the recognition of foreign molecules through STING and also augments RIG-I and TLR signalling, which leads to a marked interferon response. It is possible that introducing cGAMP into cells through EVs may trigger an antiviral response in neighbouring cells ${ }^{152}$. This represents perhaps the most direct demonstration that cellular EVs can have antiviral and pro-host roles.

The HSV-1 viral miRNAs miR-H28 and miR-H29 are also incorporated into EVs. These have been shown to have pro-viral roles in infection and facilitate the infection of neighbouring cells ${ }^{153}$, perhaps by weakening innate immune defences. Further studies are required to fully understand this observation, but it seems counterintuitive for the same infected cell to secrete pro-viral and antiviral EVs at the same time. More likely, these distinct effects that were identified in cultured cell lines represent different steps in natural infection, perhaps by altering the initial infection of different cell types, enhancing or limiting systemic spread throughout the host, or by altering viral virulence to facilitate persistence or the establishment of latency. In summary, herpesviruses, by virtue of their large genomes (encoding $>100$ proteins, as well as miRNAs and long non-coding RNAs $(\operatorname{lncRNAs}))^{154}$, modulate the biogenesis and function of EVs through several independent mechanisms.

\section{Summary and outlook}

In this Review, we have discussed the multiple ways in which different viruses manipulate EVs for their benefit to increase their persistence, pathogenesis and transmission. In recent years, EVs have emerged as specific carriers of cellular and viral components, including miRNAs, proteins and viral genomes. This can happen during active viral replication or during viral latency. The majority of experiments that have been carried out have explored how EVs can deliver cytoplasmic contents from one cell to other cells in the surrounding environment in the absence of cell-cell fusion. However, the role of EVs can also be more far-reaching than the local environment 


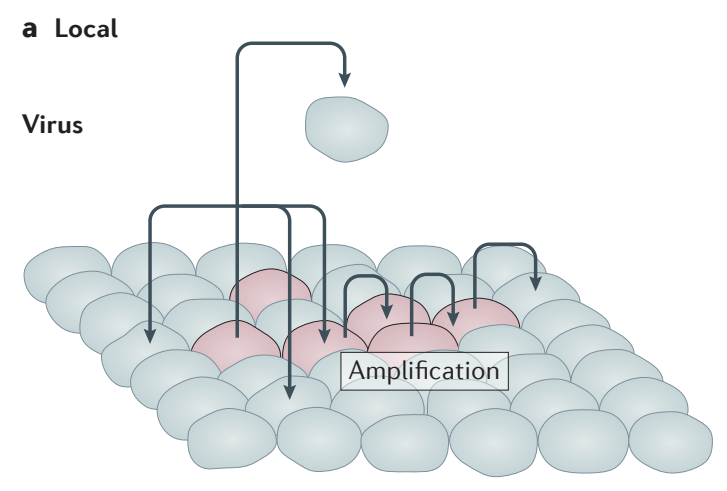

b Systemic
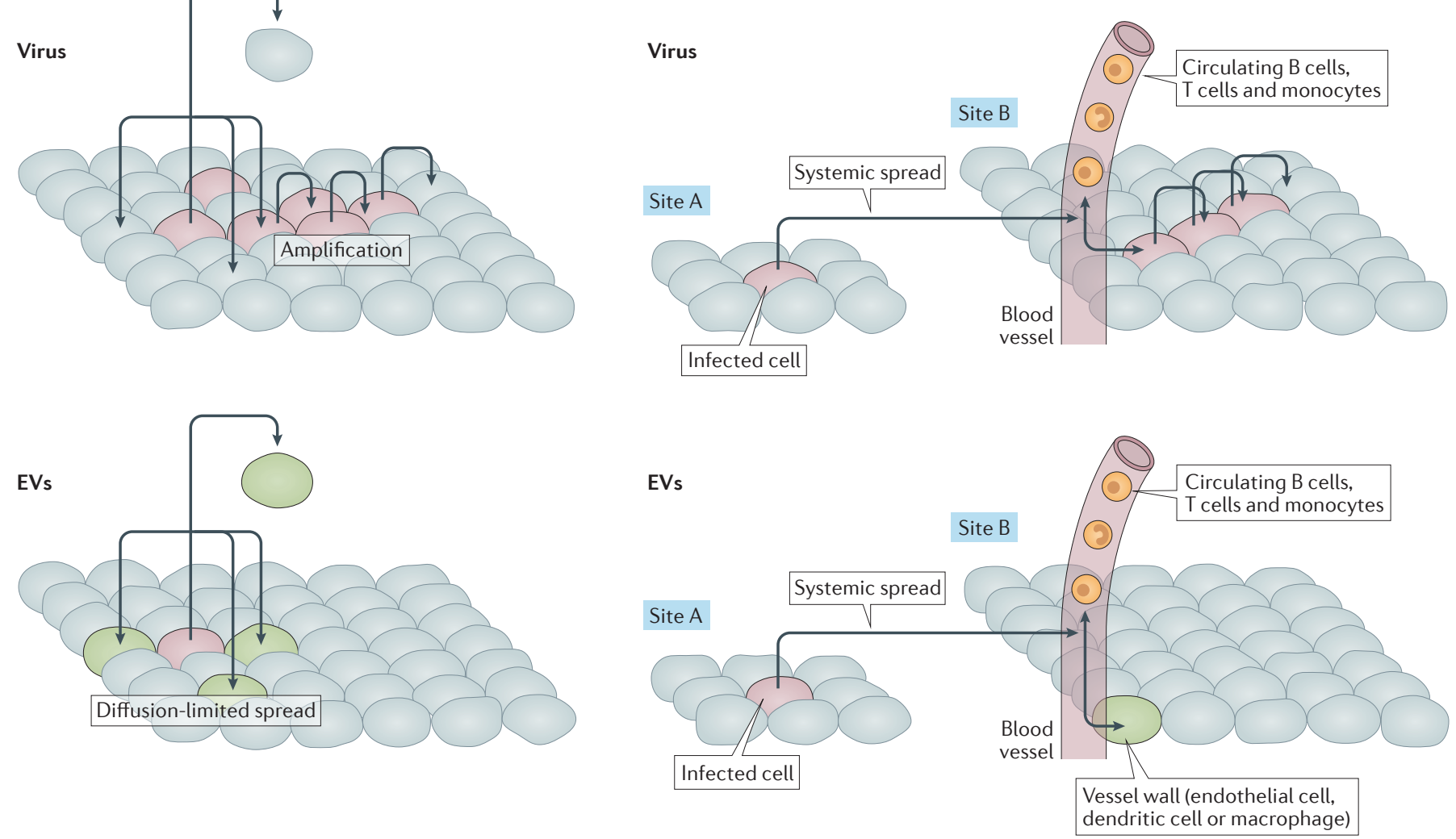

Figure 4 | Local and systemic spread of viruses and EVs. a | In a tissue or microenvironment, viruses are amplified in each permissive cell and the number of infected cells increases over time. When EVs are transported to cells, no amplification takes place and therefore any phenotype caused by the uptake of EVs is diffusion-limited. $\mathbf{b}$ |Viruses and extracellular vesicles (EVs) can spread systemically from one site (site A) to another (site B) by infecting and transmitting cells that circulate in the blood, lymph or interstitial fluids. For EVs, during systemic spread only cells that are directly exposed to EVs in the circulation, which are mostly endothelial cells, can be modulated by EVs. The spread of virions or EVs is indicated by black connecting arrows. Red cells indicate the amplification of viruses; green cells indicate the release of $E V s$ without subsequent amplification.

into which they are released (FIG. 4); the presence of EVs in the blood and the lymphatic system suggests that EVs are able to transport cargo over long distances.

The role of EVs in the pathogenesis of viruses is similar to their role in cancer metastasis, in which they are known to prime distant sites (soil) for the reception of metastatic cells (seed) (BOX 2). EVs and their effects on recipient cells are mediated by individual EVs, and the recipient cells do not produce or amplify these EVs. This is in contrast to cells infected by viruses, which can produce thousands of progeny from a single infected cell (FIG. 4). However, during chronic or latent viral infection, a single infected cell may actually release many more EVs than infectious virus particles; therefore, the use of EVs to prime and enhance systemic viral infection is likely.

Experimental data that support a role for EVs in priming the innate immune response have been reported. Cargo in EVs can elicit a TLR-dependent immune response in mice that have tumours ${ }^{155}$ or prime neighbouring cells, including DCs, to respond to viral infection by priming the adaptive immune response or through the release of interferon ${ }^{134,156}$. During this scenario, EVs may have evolved a role for protection from pathogens, perhaps through the activation of innate immune responses in neighbouring cells. A role for EVs in adaptive immunity has already been established, in which EVs (or some specialized membrane-encapsulated vesicles) can efficiently cross-prime innate immunity and adaptive immune memory responses ${ }^{27,28,30,101,157}$.

Importantly, it is likely that basic research in the biogenesis and fusion of EVs will be enhanced by previous, similar studies that characterized virion egress and entry. Many stages in the biogenesis of EVs are also used by viruses; for example, the ESCRT family of proteins during virus assembly and egress. Further study of viral effects on the pathways that involve EVs is likely to identify the crucial regulators of endosomal and exosomal trafficking in host cell physiology, as well as uncover new mechanisms that modulate host cells to determine the outcome of virus infections. Further study of the biology of EVs will provide a rich area to enhance our understanding of the complexities of viral redirection of cellular processes and the determination of how viral effects on the production and content of EVs contribute to both pathogenesis and persistence. 
1. Raposo, G. \& Stoorvogel, W. Extracellular vesicles: exosomes, microvesicles, and friends. J. Cell Biol. 200 373-383 (2013)

2. Kowal, J., Tkach, M. \& Thery, C. Biogenesis and secretion of exosomes. Curr. Opin. Cell Biol. 29, 116-125 (2014)

This study provides a comprehensive review of the contribution of distinct vesicle transport proteins to the formation and secretion of exosomes.

3. Knipe, D. M. \& Howley, P. M. Fields Virology 6th edn (Wolters Kluwer/Lippincott Williams \& Wilkins Health, 2013).

4. Freed, E. O. HIV-1 assembly, release and maturation. Nat. Rev. Microbiol. 13, 484-496 (2015).

5. Feng, Z. et al. A pathogenic picornavirus acquires an envelope by hijacking cellular membranes. Nature 496, 367-371 (2013).

This study is the first to show that a non-enveloped virus can acquire EV membranes and remain infectious after being released from infected cells using the ESCRT complex.

6. Hirai-Yuki, A., Hensley, L., Whitmire, J. K. $\&$ Lemon, S. M. Biliary secretion of quasi-enveloped human hepatitis A virus. mBio 7, e01998-16 (2016)

7. Chen, Y. H. et al. Phosphatidylserine vesicles enable efficient en bloc transmission of enteroviruses. Cell 160, 619-630 (2015) This study demonstrates that many virus particles can be incorporated into large microvesicles.

8. Munz, C. The autophagic machinery in viral exocytosis Front. Microbiol. 8, 269 (2017).

9. Robinson, S. M. et al. Coxsackievirus B exits the host cell in shed microvesicles displaying autophagosomal markers. PLoS Pathog. 10, e1004045 (2014).

10. Meckes, D. G. Jr \& Raab-Traub, N. Microvesicles and viral infection. J. Virol. 85, 12844-12854 (2011).

11. Spear, P. G. \& Roizman, B. Proteins specified by herpes simplex virus. V. Purification and structural proteins of the herpesvirion. J. Virol. 9, 143-159 (1972).

12. Kowal, J. et al. Proteomic comparison defines novel markers to characterize heterogeneous populations of extracellular vesicle subtypes. Proc. Natl Acad. Sci. USA 113, E968-E977 (2016).

This study provides evidence for different populations of EVs circulating at the same time, with each population being identified by a different composition of surface markers.

13. Chugh, P. E. et al. Systemically circulating viral and tumor-derived microRNAs in KSHV-associated malignancies. PLoS Pathog. 9, e1003484 (2013). This study reveals the secretion of host and vira miRNAs within EVs in the sera of patients and in mouse models, and shows that the uptake of these EVs modulates cell behaviour and induces cell migration and the secretion of IL-6.

14. Cliffe, A. R., Nash, A. A. \& Dutia, B. M. Selective uptake of small RNA molecules in the virion of murine gammaherpesvirus 68. J. Virol. 83, 2321-2326 (2009).

15. Amen, M. A. \& Griffiths, A. Packaging of non-coding RNAs into herpesvirus virions: comparisons to coding RNAs. Front. Genet. 2, 81 (2011)

16. Meckes, D. G. Jr et al. Human tumor virus utilizes exosomes for intercellular communication. Proc. Nat Acad. Sci. USA 107, 20370-20375 (2010). This study is the first to identify the biological properties of EVs produced by cells that express the viral oncogene LMP1, and shows that the uptake of EVs results in the activation of ERK and AKT signalling pathways.

17. Bihrer, V. et al. Serum miR-122 as a biomarker of necroinflammation in patients with chronic hepatitis $C$ virus infection. Am. J. Gastroenterol. 106, 1663-1669 (2011).

18. Xu, J. et al. Circulating microRNAs, miR-21, miR-122, and miR-223, in patients with hepatocellular carcinoma or chronic hepatitis. Mol. Carcinog. 50, 136-142 (2011)

19. Laterza, O F et al. Plasma microRNAs as sensitive and specific biomarkers of tissue injury. Clin. Chem. 55, 1977-1983 (2009)

20. Lanford, R. E. et al. Therapeutic silencing of microRNA-122 in primates with chronic hepatitis C virus infection. Science 327, 198-201 (2010).

21. Jopling, C. L., Yi, M., Lancaster, A. M., Lemon, S. M. \& Sarnow, P. Modulation of hepatitis C virus RNA abundance by a liver-specific microRNA. Science 309 1577-1581 (2005).

22. Masaki, T. et al. miR-122 stimulates hepatitis $C$ virus RNA synthesis by altering the balance of viral RNAs engaged in replication versus translation. Cell Host Microbe 17, 217-228 (2015)

23. Gould, S. J., Booth, A. M. \& Hildreth, J. E. The Trojan exosome hypothesis. Proc. Natl Acad. Sci. USA 100, 10592-10597 (2003)

This paper proposes the Trojan horse hypothesis for hiding viruses and viral components inside EVs.

24. Pegtel, D. M. et al. Functional delivery of viral miRNAs via exosomes. Proc. Natl Acad. Sci. USA 107 6328-6333 (2010)

This study reports the delivery of viral miRNAs into target cells through EVs.

25. Sowinski, S. et al. Membrane nanotubes physically connect $T$ cells over long distances presenting a nove route for HIV-1 transmission. Nat. Cell Biol. 10, 211-219 (2008).

26. Karlikow, M. et al. Drosophila cells use nanotube-like structures to transfer dsRNA and RNAi machinery between cells. Sci. Rep. 6, 27085 (2016).

27. Xu, W. et al. HIV-1 evades virus-specific IgG2 and IgA responses by targeting systemic and intestinal B cells via long-range intercellular conduits. Nat. Immunol. 10, 1008-1017 (2009).

28. Wolfers, J. et al. Tumor-derived exosomes are a source of shared tumor rejection antigens for CTL crosspriming. Nat. Med. 7, 297-303 (2001). This study reports evidence for the role of EVs in antigenic cross-priming

29. Arrode, G., Boccaccio, C., Abastado, J. P. \& Davrinche, $C$. Cross-presentation of human cytomegalovirus pp65 (UL83) to CD8 ${ }^{+} \mathrm{T}$ cells is regulated by virus-induced, soluble-mediatordependent maturation of dendritic cells. J. Virol. 76 142-150 (2002)

30. Joffre, O. P., Segura, E., Savina, A. \& Amigorena, S. Cross-presentation by dendritic cells. Nat. Rev. Immunol. 12, 557-569 (2012)

31. Mori, Y. et al. Human herpesvirus- 6 induces MVB formation, and virus egress occurs by an exosomal release pathway. Traffic 9, 1728-1742 (2008).

32. Lenassi, M. et al. HIV Nef is secreted in exosomes and triggers apoptosis in bystander CD4 ${ }^{+} \mathrm{T}$ cells. Traffic 11, 110-122 (2010)

33. Lee, J. H. et al. HIV-Nef and ADAM17-containing plasma extracellular vesicles induce and correlate with immune pathogenesis in chronic HIV infection. EBioMedicine 6, 103-113 (2016)

34. Raymond, A. D. et al. HIV type $1 \mathrm{Nef}$ is released from infected cells in CD45+ microvesicles and is present in the plasma of HIV-infected individuals. AIDS Res. Hum. Retroviruses 27, 167-178 (2011)

35. Caby, M. P., Lankar, D., Vincendeau-Scherrer, C. Raposo, G. \& Bonnerot, C. Exosomal-like vesicles are present in human blood plasma. Int. Immunol. 17 879-887 (2005).

36. Nolte-'t Hoen, E., Cremer, T., Gallo, R. C. \& Margolis, L. B. Extracellular vesicles and viruses: are they close relatives? Proc. Natl Acad. Sci. USA 113, 9155-9161 (2016)

37. Meckes, D. G. Jr. Exosomal communication goes viral. J. Virol. 89, 5200-5203 (2015).

38. Longatti, A. The dual role of exosomes in hepatitis A and $C$ virus transmission and viral immune activation. Viruses 7, 6707-6715 (2015).

39. Hergenreider, $\mathrm{E}$ et al. Atheroprotective communication between endothelial cells and smooth muscle cells through miRNAs. Nat. Cell Biol. 14, 249-256 (2012).

40. Gibbings, D. J., Ciaudo, C., Erhardt, M. \& Voinnet, O. Multivesicular bodies associate with components of miRNA effector complexes and modulate miRNA activity. Nat. Cell Biol. 11, 1143-1149 (2009).

41. Kosaka, N. et al. Secretory mechanisms and intercellular transfer of microRNAs in living cells. J. Biol. Chem. 285, 17442-17452 (2010)

42. Mathivanan, S., Fahner, C. J., Reid, G. E. \& Simpson, R. J. ExoCarta 2012: database of exosomal proteins, RNA and lipids. Nucleic Acids Res. 40 D1241-D1244 (2012).

This study provides details of a database of proteins and RNAs that have been reported to be associated with EVs.

43. Mittelbrunn, M. et al. Unidirectional transfer of microRNA-loaded exosomes from $T$ cells to antigenpresenting cells. Nat. Commun. 2, 282 (2011).

44. Koppers-Lalic, D. et al. Nontemplated nucleotid additions distinguish the small RNA composition in cells from exosomes. Cell Rep. 8, 1649-1658 (2014).

45. Lunavat, T. R. et al. Small RNA deep sequencing discriminates subsets of extracellular vesicles released by melanoma cells - evidence of unique microRNA cargos. RNA Biol. 12, 810-823 (2015).
46. Kharkwal, H. Smith, C. G. \& Wilson, D. W. Herpes simplex virus capsid localization to ESCRT-VPS4 complexes in the presence and absence of the large tegument protein UL36p. J. Virol. 90, 7257-7267 (2016)

47. Verweij, F. J. et al. Exosomal sorting of the viral oncoprotein LMP1 is restrained by TRAF2 association at signalling endosomes. J. Extracell. Vesicles 4 26334 (2015)

48. Hurwitz, S. N. et al. CD63 regulates Epstein-Barr virus LMP1 exosomal packaging, enhancement of vesicle production, and noncanonical NF-KB signaling. J. Virol. 91, e02251-16 (2017)

49. Wilson, C. M. et al. Sortilin mediates the release and transfer of exosomes in concert with two tyrosine kinase receptors. J. Cell Sci. 127, 3983-3997 (2014).

50. Roucourt, B., Meeussen, S., Bao, J., Zimmermann, P. $\&$ David, G. Heparanase activates the syndecansyntenin-ALIX exosome pathway. Cell Res. 25 412-428 (2015)

51. Ghossoub, R. et al. Syntenin-ALIX exosome biogenesis and budding into multivesicular bodies are controlled by ARF6 and PLD2. Nat. Commun. 5, 3477 (2014).

52. Ostrowski, M. et al. Rab27a and Rab27b control different steps of the exosome secretion pathway. Nat. Cell Biol. 12, 19-30 (2010) This study demonstrates a role for specific GTPase in the regulation of EVs.

53. Fotheringham, J. A. \& Raab-Traub, N. Epstein-Barr virus latent membrane protein 2 induces autophagy to promote abnormal acinus formation. J. Virol. 89 6940-6944 (2015).

54. Inoue, J. et al. HBV secretion is regulated through the activation of endocytic and autophagic compartments mediated by Rab7 stimulation. J. Cell Sci. 128 1696-1706 (2015).

55. Johns, H. L., Gonzalez-Lopez, C., Sayers, C. L., Hollinshead, M. \& Elliott, G. Rab6 dependent postGolgi trafficking of HSV1 envelope proteins to sites of virus envelopment. Traffic 15, 157-178 (2014).

56. Veettil, M. V. et al. ESCRT-0 component Hrs promotes macropinocytosis of Kaposi's sarcoma-associated herpesvirus in human dermal microvascular endothelial cells. J. Virol. 90, 3860-3872 (2016).

57. Hyenne, V. et al. RAL-1 controls multivesicular body biogenesis and exosome secretion. J. Cell Biol. 211 27-37 (2015)

58. Cruz, L. et al. Potent inhibition of human cytomegalovirus by modulation of cellular SNARE syntaxin 5. J. Virol. 91, e01637-16 (2017).

59. Elgner, F. et al. Characterization of $\alpha$-taxilin as a novel factor controlling the release of hepatitis $C$ virus. Biochem. J. 473, 145-155 (2016)

60. Kawabata, A., Serada, S., Naka, T. \& Mori, Y. Human herpesvirus $6 \mathrm{gM} / \mathrm{gN}$ complex interacts with $\mathrm{v}$-SNARE in infected cells. J. Gen. Virol. 95, 2769-2777 (2014).

61. Vallhov, H. et al. Exosomes containing glycoprotein 350 released by EBV-transformed $B$ cells selectively target B cells through CD21 and block EBV infection in vitro. J. Immunol. 186, 73-82 (2011).

62. Fitzner, D. et al. Selective transfer of exosomes from oligodendrocytes to microglia by macropinocytosis. J. Cell Sci. 124, 447-458 (2011).

63. van Dongen, H. M., Masoumi, N., Witwer, K. W. $\delta$ Pegtel, D. M. Extracellular vesicles exploit viral entry routes for cargo delivery. Microbiol. Mol. Biol. Rev. 80, 369-386 (2016).

64. Ruiss, R., Jochum, S., Mocikat, R. Hammerschmidt, W. \& Zeidler, R. EBV-gp350 confers B-cell tropism to tailored exosomes and is a neoantigen in normal and malignant B cells - a new option for the treatment of B-CLL. PLOS ONE 6 e25294 (2011)

65. Shukla, D. \& Spear, P. G. Herpesviruses and heparan sulfate: an intimate relationship in aid of viral entry. J. Clin. Invest. 108, 503-510 (2001).

66. Christianson, H. C., Svensson, K. J., van Kuppevelt, T. H., Li, J. P. \& Belting, M. Cancer cell exosomes depend on cell-surface heparan sulfate proteoglycans for their internalization and functional activity. Proc. Natl Acad. Sci. USA 110, 17380-17385 (2013)

67. Koumangoye, R. B., Sakwe, A. M., Goodwin, J. S., Patel, T. \& Ochieng, J. Detachment of breast tumor cells induces rapid secretion of exosomes which subsequently mediate cellular adhesion and spreading. PLOS ONE 6, e24234 (2011).

68. Sung, B. H., Ketova, T., Hoshino, D., Zijlstra, A. \& Weaver, A. M. Directional cell movement through tissues is controlled by exosome secretion. Nat. Commun. 6, 7164 (2015). 
69. Lee, H. D., Koo, B. H., Kim, Y. H., Jeon, O. H. \& Kim, D. S. Exosome release of ADAM 15 and the functional implications of human macrophage-derived ADAM 15 exosomes. FASEB J. 26, 3084-3095 (2012)

70. Akula, S. M., Pramod, N. P., Wang, F. Z. \& Chandran, B. Integrin $\alpha 3 \beta 1$ (CD 49c/29) is a cellular receptor for Kaposi's sarcoma-associated herpesvirus (KSHV/HHV-8) entry into the target cells. Cell 108, 407-419 (2002).

71. Zheng, X. et al. Treatment with hyperimmune equine immunoglobulin or immunoglobulin fragments completely protects rodents from Ebola virus infection. Sci. Rep. 6, 24179 (2016)

72. de Gassart, A., Geminard, C., Fevrier, B., Raposo, G. $\&$ Vidal, M. Lipid raft-associated protein sorting in exosomes. Blood 102, 4336-4344 (2003).

73. Peinado, H. et al. Melanoma exosomes educate bone marrow progenitor cells toward a pro-metastatic phenotype through MET. Nat. Med. 18, 883-891 (2012).

74. Ono, M. et al. Exosomes from bone marrow mesenchymal stem cells contain a microRNA that promotes dormancy in metastatic breast cancer cells. Sci. Signal. 7, ra63 (2014)

75. Atay, S. et al. Oncogenic KIT-containing exosomes increase gastrointestinal stromal tumor cell invasion. Proc. Natl Acad. Sci. USA 111, 711-716 (2014).

76. Zhou, W. et al. Cancer-secreted miR-105 destroys vascular endothelial barriers to promote metastasis. Cancer Cell 25, 501-515 (2014).

77. Catanese, M. T. et al. Ultrastructural analysis of hepatitis C virus particles. Proc. Natl Acad. Sci. USA 110, 9505-9510 (2013)

78. Xiao, F. et al. Hepatitis C virus cell-cell transmission and resistance to direct-acting antiviral agents. PLoS Pathog. 10, e1004128 (2014). This study shows the direct cell-to-cell transfer of HCV that was resistant to antivirals but could be sensitized using entry inhibitors such as claudin antibody.

79. Ramakrishnaiah, V. et al. Exosome-mediated transmission of hepatitis $\mathrm{C}$ virus between human hepatoma Huh7.5 cells. Proc. Natl Acad. Sci. USA 110, 13109-13113 (2013)

80. Dreux, M. et al. Short-range exosomal transfer of viral RNA from infected cells to plasmacytoid dendritic cells triggers innate immunity. Cell Host Microbe 12, 558-570 (2012)

81. Masciopinto, F. et al. Association of hepatitis C virus envelope proteins with exosomes. Eur. J. Immunol. 34, 2834-2842 (2004).

82. Zhang, J. et al. CD81 is required for hepatitis $C$ virus glycoprotein-mediated viral infection. J. Virol. 78 1448-1455 (2004).

83. von Schaewen, M. et al. Expanding the host range of hepatitis $\mathrm{C}$ virus through viral adaptation. $m B$ Bio 7 , e01915-16 (2016)

84. Pileri, P. et al. Binding of hepatitis $C$ virus to CD81 Science 282, 938-941 (1998).

85. Brimacombe, C. L. et al. Neutralizing antibodyresistant hepatitis $\mathrm{C}$ virus cell-to-cell transmission. J. Virol. 85, 596-605 (2011).

86. Inal, J. M. \& Jorfi, S. Coxsackievirus B transmission and possible new roles for extracellular vesicles. Biochem. Soc. Trans. 41, 299-302 (2013).

87. Mao, L. et al. Enterovirus 71 transmission by exosomes establishes a productive infection in human neuroblastoma cells. Virus Genes 52, 189-194 (2016).

88. Brenchley, J. M. et al. Microbial translocation is a cause of systemic immune activation in chronic HIV infection. Nat. Med. 12, 1365-1371 (2006).

89. Johnson, T. P. et al. Induction of IL-17 and nonclassical T-cell activation by HIV-Tat protein. Proc. Natl Acad. Sci. USA 110, 13588-13593 (2013)

90. Izquierdo-Useros, N. et al. HIV and mature dendritic cells: Trojan exosomes riding the Trojan horse? PLoS Pathog. 6, e1000740 (2010).

91. Fang, Y. et al. Higher-order oligomerization targets plasma membrane proteins and HIV gag to exosomes. PLoS Biol. 5, e158 (2007)

92. Nguyen, D. G., Booth, A., Gould, S. J. \& Hildreth, J. E. Evidence that HIV budding in primary macrophages occurs through the exosome release pathway. J. Biol. Chem. 278, 52347-52354 (2003).

93. Doitsh, G. et al. Abortive HIV infection mediates CD4 $\mathrm{T}$ cell depletion and inflammation in human lymphoid tissue. Cell 143, 789-801 (2010).

94. Kadiu, I., Narayanasamy, P., Dash, P. K., Zhang, W. $\&$ Gendelman, H. E. Biochemical and biologic characterization of exosomes and microvesicles as facilitators of HIV-1 infection in macrophages. J. Immunol. 189, 744-754 (2012).

95. Izquierdo-Useros, N. et al. Capture and transfer of HIV-1 particles by mature dendritic cells converges with the exosome-dissemination pathway. Blood 113 2732-2741 (2009)

96. Wiley, R. D. \& Gummuluru, S. Immature dendritic cellderived exosomes can mediate HIV-1 trans infection. Proc. Natl Acad. Sci. USA 103, 738-743 (2006).

97. Li, M. et al. Quantitative proteomic analysis of exosomes from HIV-1-infected lymphocytic cells. Proteomics 12, 2203-2211 (2012)

98. Sheehy, A. M., Gaddis, N. C., Choi, J. D. \& Malim, M. H. Isolation of a human gene that inhibits HIV-1 infection and is suppressed by the viral Vif protein. Nature 418, 646-650 (2002).

99. Tumne, A. et al. Noncytotoxic suppression of human immunodeficiency virus type 1 transcription by exosomes secreted from CD8 ${ }^{+}$T cells. J. Virol. 83 4354-4364 (2009).

100. Andre, F. et al. Exosomes as potent cell-free peptidebased vaccine. I. Dendritic cell-derived exosomes transfer functional MHC class 1/peptide complexes to dendritic cells. J. Immunol. 172, 2126-2136 (2004).

101. Kovar, M. et al. Direct stimulation of T cells by membrane vesicles from antigen-presenting cells. Proc. Natl Acad. Sci. USA 103, 11671-11676 (2006)

102. Gaulke, C. A. et al. Intestinal epithelial barrier disruption through altered mucosal microRNA expression in human immunodeficiency virus and simian immunodeficiency virus infections. J. Virol. 88, 6268-6280 (2014).

103. Chang, S. T. et al. Next-generation sequencing of small RNAs from HIV-infected cells identifies phased microrna expression patterns and candidate novel microRNAs differentially expressed upon infection. mBio 4, e00549-12 (2013).

104. Triboulet, R. et al. Suppression of microRNA-silencing pathway by HIV-1 during virus replication. Science 315, 1579-1582 (2007)

105. Thapa, D. R. et al. Serum microRNAs in HIV-infected individuals as pre-diagnosis biomarkers for AIDS-NHL. J. Acquir. Immune Defic. Syndr. 66, 229-237 (2014).

106. Narayanan, A. et al. Exosomes derived from HIV-1-infected cells contain trans-activation response element RNA. J. Biol. Chem. 288, 20014-20033 (2013)

107. Whisnant, A. W. et al. In-depth analysis of the interaction of HIV-1 with cellular microRNA biogenesis and effector mechanisms. $\mathrm{mBio} 4$ e000193 (2013)

108. Cullen, B. R., Cherry, S. \& tenOever, B. R. Is RNA interference a physiologically relevant innate antiviral immune response in mammals? Cell Host Microbe 14 374-378 (2013)

109. Kincaid, R. P., Chen, Y., Cox, J. E., Rethwilm, A. \& Sullivan, C. S. Noncanonical microRNA (miRNA) biogenesis gives rise to retroviral mimics of lymphoproliferative and immunosuppressive host miRNAs. mBio 5, e00074 (2014).

110. Whisnant, A. W. et al. Identification of novel, highly expressed retroviral microRNAs in cells infected by bovine foamy virus. J. Virol. 88, 4679-4686 (2014).

11. Kincaid, R. P., Burke, J. M. \& Sullivan, C. S. RNA virus microRNA that mimics a B-cell oncomiR. Proc. Natl Acad. Sci. USA 109, 3077-3082 (2012).

112. Nolte-'t Hoen, E. N. et al. Deep sequencing of RNA from immune cell-derived vesicles uncovers the selective incorporation of small non-coding RNA biotypes with potential regulatory functions. Nucleic Acids Res. 40, 9272-9285 (2012).

113. Pare, J. M. \& Sullivan, C. S. Distinct antiviral responses in pluripotent versus differentiated cells PLoS Pathog. 10, e 1003865 (2014).

114. Sufiawati, I. \& Tugizov, S. M. HIV-associated disruption of tight and adherens junctions of oral epithelial cells facilitates HSV-1 infection and spread. PLOS ONE 9, e88803 (2014)

115. Ensoli, B. et al. Synergy between basic fibroblast growth factor and HIV-1 Tat protein in induction of Kaposi's sarcoma. Nature 371, 674-680 (1994).

116. Debaisieux, S., Rayne, F., Yezid, H. \& Beaumelle, B. The ins and outs of HIV-1 Tat. Traffic 13, 355-363 (2012)

117. Wender, P. A. et al. The design, synthesis, and evaluation of molecules that enable or enhance cellular uptake: peptoid molecular transporters. Proc. Natl Acad. Sci. USA 97, 13003-13008 (2000).
118. Nagahara, H. et al. Transduction of full-length TAT fusion proteins into mammalian cells: TAT-p27Kip 1 induces cell migration. Nat. Med. 4, 1449-1452 (1998).

119. Amorim, N. A. et al. Interaction of HIV-1 Nef protein with the host protein Alix promotes lysosomal targeting of CD4 receptor. J. Biol. Chem. 289, 27744-27756 (2014).

120. Dikeakos, J. D. et al. An interdomain binding site on HIV-1 Nef interacts with PACS- 1 and PACS- 2 on endosomes to down-regulate MHC-I. Mol. Biol. Cell 23, 2184-2197 (2012).

121. Aqil, M. et al. The HIV Nef protein modulates cellular and exosomal miRNA profiles in human monocytic cells. J. Extracell. Vesicles http://dx.doi.org/10.3402/ jev.v3.23129 (2014).

122. Luo, X., Fan, Y., Park, I. W. \& He, J. J. Exosomes are unlikely involved in intercellular Nef transfer. PLoS ONE 10, e0124436 (2015)

123. Flanagan, J., Middeldorp, J. \& Sculley, T. Localization of the Epstein-Barr virus protein LMP1 to exosomes. J. Gen. Virol. 84, 1871-1879 (2003)

124. Raab-Traub, N. in Human Herpesviruses: Biology, Therapy, and Immunoprophylaxis (eds Arvin, A. et al.) 986-1006 (Cambridge Univ. Press, 2007).

125. Houali, K. et al. A new diagnostic marker for secreted Epstein-Barr virus encoded LMP1 and BARF1 oncoproteins in the serum and saliva of patients with nasopharyngeal carcinoma. Clin. Cancer Res. 13 4993-5000 (2007)

126. Verweij, F. J. et al. LMP1 association with CD63 in endosomes and secretion via exosomes limits constitutive NF- $\mathrm{kB}$ activation. EMBO J. 30 2115-2129 (2011)

127. Ardila-Osorio, H. et al. TRAF interactions with raft-like buoyant complexes, better than TRAF rates of degradation, differentiate signaling by CD40 and EBV latent membrane protein 1. Int. J. Cancer 113 267-275 (2005)

128. Dukers, D. F. et al. Direct immunosuppressive effects of EBV-encoded latent membrane protein 1. J. Immunol. 165, 663-670 (2000).

129. Klibi, J. et al. Blood diffusion and Th1-suppressive effects of galectin-9-containing exosomes released by Epstein-Barr virus-infected nasopharyngeal carcinoma cells. Blood 113, 1957-1966 (2009).

130. Keryer-Bibens, C. et al. Exosomes released by EBVinfected nasopharyngeal carcinoma cells convey the viral latent membrane protein 1 and the immunomodulatory protein galectin 9. BMC Cancer 6 , 283 (2006).

131. Meckes, D. G. Jr et al. Modulation of B-cell exosome proteins by gamma herpesvirus infection. Proc. Nat Acad. Sci. USA 110, E2925-E2933 (2013).

This study reports a comprehensive proteomic analysis of EVs that are produced by B cell lines infected with EBV, KSHV, or EBV and KSHV, which led to the prediction that EVs can modulate cell death and survival.

132. Ceccarelli, S. et al. Epstein-Barr virus latent membrane protein 1 promotes concentration in multivesicular bodies of fibroblast growth factor 2 and its release through exosomes. Int. J. Cancer 121 , 1494-1506 (2007).

133. Aga, M. et al. Exosomal HIF1 $\alpha$ supports invasive potential of nasopharyngeal carcinoma-associated LMP1-positive exosomes. Oncogene 33, 4613-4622 (2014).

134. Baglio, S. R. et al. Sensing of latent EBV infection through exosomal transfer of 5'pppRNA. Proc. Nat/ Acad. Sci. USA 113, E587-E596 (2016)

135. Cavallin, L. E., Goldschmidt-Clermont, P. \& Mesri, E. A Molecular and cellular mechanisms of KSHV oncogenesis of Kaposi's sarcoma associated with HIV/AIDS. PLoS Pathog. 10, e1004154 (2014).

136. Cai, X. et al. Kaposi's sarcoma-associated herpesvirus expresses an array of viral microRNAs in latently infected cells. Proc. Natl Acad. Sci. USA 102 5570-5575 (2005).

137. Samols, M. A., Hu, J., Skalsky, R. L \& Renne, R. Cloning and identification of a microRNA cluster within the latency-associated region of Kaposi's sarcomaassociated herpesvirus. J. Virol. 79, 9301-9305 (2005).

138. O'Hara, A. J. et al. Pre-micro RNA signatures delineate stages of endothelial cell transformation in Kaposi sarcoma. PLoS Pathog. 5, e1000389 (2009).

139. Lin, X., Li, X., Liang, D. \& Lan, K. MicroRNAs and unusual small RNAs discovered in Kaposi's sarcomaassociated herpesvirus virions. J. Virol. $\mathbf{8 6}$ 12717-12730 (2012). 
140. Chugh, P., Tamburro, K. \& Dittmer, D. P. Profiling of premicro RNAs and microRNAs using quantitative realtime PCR (qPCR) arrays. J. Vis. Exp. http://dx.doi.org/ $10.3791 / 2210$ (2010)

141. Myoung, J. \& Ganem, D. Generation of a doxycyclineinducible KSHV producer cell line of endothelial origin: maintenance of tight latency with efficient reactivation upon induction. J. Virol. Methods 174 , 12-21 (2011).

142. Rainy, N., Zayoud, M., Kloog, Y., Rechavi, O. \& Goldstein, I. Viral oncomiR spreading between $B$ and T cells is employed by Kaposi's sarcoma herpesvirus to induce non-cell-autonomous target gene regulation. Oncotarget 7, 41870-41884 (2016).

143. Singh, V. V. et al. Kaposi's sarcoma-associated herpesvirus latency in endothelial and B cells activates gamma interferon-inducible protein 16-mediated inflammasomes. J. Virol. 87 4417-4431 (2013).

144. Sullivan, B. M. \& Coscoy, L. The U24 protein from human herpesvirus 6 and 7 affects endocytic recycling. J. Virol. 84, 1265-1275 (2010).

145. Temme, S., Eis-Hubinger, A. M., McLellan, A. D. \& Koch, $\mathrm{N}$. The herpes simplex virus- 1 encoded glycoprotein B diverts HLA-DR into the exosome pathway. J. Immunol. 184, 236-243 (2010).

146. Hogue, I. B., Scherer, J. \& Enquist, L. W. Exocytosis of alphaherpesvirus virions, light particles, and glycoproteins uses constitutive secretory mechanisms. mBio 7, e00820-16 (2016).

147. Tandon, R., AuCoin, D. P. \& Mocarski, E. S Human cytomegalovirus exploits ESCRT machinery in the process of virion maturation. J. Virol. 83, 10797-10807 (2009).

148. Cepeda, V., Esteban, M. \& Fraile-Ramos, A. Human cytomegalovirus final envelopment on membranes containing both trans-Golgi network and endosomal markers. Cell. Microbiol. 12, 386-404 (2010).

149. Walker, J. D., Maier, C. L. \& Pober, J. S

Cytomegalovirus-infected human endothelial cells can stimulate allogeneic $\mathrm{CD}^{+}$memory $\mathrm{T}$ cells by releasing antigenic exosomes. J. Immunol. 182, 1548-1559 (2009).
150. Kalamvoki, M., Du, T. \& Roizman, B. Cells infected with herpes simplex virus 1 export to uninfected cells exosomes containing STING, viral mRNAs, and microRNAs. Proc. Natl Acad. Sci. USA 111, E4991-E4996 (2014).

This study demonstrates that STING, an innate immune sensor, as well as viral mRNAs and host miRNAs are transferred from virus-infected cells to neighbouring cells without being transmitted by a virus.

151. Kalamvoki, M. \& Deschamps, T. Extracellular vesicles during herpes simplex virus type 1 infection: an inquire. Virol. J. 13, 63 (2016).

152. Bridgeman, A. et al. Viruses transfer the antiviral second messenger cGAMP between cells. Science 349, 1228-1232 (2015).

This study demonstrates that a small molecule that is involved in innate immune sensing is transferred by virions.

153. Han, Z. et al. miR-H28 and miR-H29 expressed late in productive infection are exported and restrict HSV-1 replication and spread in recipient cells. Proc. Natl Acad. Sci. USA 113, E894-E901 (2016).

154. Schifano, J. M., Corcoran, K., Kelkar, H. \& Dittmer, D. P. Expression of the antisense-to-latency transcript long noncoding RNA in Kaposi's sarcomaassociated herpesvirus. J. Virol. 91, e01698-16 (2017)

155. Fabbri, M. et al. MicroRNAs bind to Toll-like receptors to induce prometastatic inflammatory response. Proc. Natl Acad. Sci. USA 109, E2110-E2116 (2012).

156. Li, J. et al. Exosomes mediate the cell-to-cell transmission of IFN- $\alpha$-induced antiviral activity. Nat. Immunol. 14, 793-803 (2013).

157. Robbins, P. D. \& Morelli, A. E. Regulation of immune responses by extracellular vesicles. Nat. Rev. Immunol. 14, 195-208 (2014).

158. Taylor, D. D. \& Shah, S. Methods of isolating extracellular vesicles impact down-stream analyses of their cargoes. Methods 87, 3-10 (2015).

159. de Menezes-Neto, A et al. Size-exclusion chromatography as a stand-alone methodology identifies novel markers in mass spectrometry analyses of plasma-derived vesicles from healthy individuals. J. Extracell. Vesicles 4, 27378 (2015)
160. Hong C. S., Funk, S., Muller, L, Boyiadzis, M \& Whiteside, T. L. Isolation of biologically active and morphologically intact exosomes from plasma of patients with cancer. J. Extracell. Vesicles 5, 29289 (2016).

161. Welton, J. L., Webber, J. P., Botos, L. A., Jones, M. \& Clayton, A. Ready-made chromatography columns for extracellular vesicle isolation from plasma. J. Extracell. Vesicles 4, 27269 (2015).

162. Lock, M. et al. Rapid, simple, and versatile manufacturing of recombinant adeno-associated viral vectors at scale. Hum. Gene Ther. 21, 1259-1271 (2010).

163. Arroyo, J. D. et al. Argonaute2 complexes carry a population of circulating microRNAs independent of vesicles in human plasma. Proc. Natl Acad. Sci. USA 108, 5003-5008 (2011).

164. Oksvold, M. P. et al. Expression of B-cell surface antigens in subpopulations of exosomes released from B-cell lymphoma cells. Clin. Ther. 36, 847-862.e1 (2014).

165. Zomer, A. et al. In vivo imaging reveals extracellular vesicle-mediated phenocopying of metastatic behavior Cell 161, 1046-1057 (2015).

166. Kalra, H. et al. Vesiclepedia: a compendium for extracellular vesicles with continuous community annotation. PLoS Biol. 10, e1001450 (2012). This study provides details of a database of proteins and RNAs that are reported to be associated with EVs.

\section{Acknowledgements}

Work in the authors' laboratories is supported by public health service grants (DA040394 and CA019014) and the University Cancer Research Fund (UCRF). The authors thank their colleagues R. McNamara, P. Chugh and B. Damania for insightful discussions.

\section{Competing interests statement}

The authors declare competing interests: see Web version for details.

\section{Publisher's note}

Springer Nature remains neutral with regard to jurisdictional claims in published maps and institutional affiliations. 